\title{
Revealing the formation histories of the first stars with the cosmic near-infrared background
}

\author{
Guochao Sun ${ }^{\circledR},{ }^{1 \star}$ Jordan Mirocha ${ }^{\circledR}, 2$ Richard H. Mebane ${ }^{\circledR 3,4}$ and Steven R. Furlanetto ${ }^{\circledR 4}$ \\ ${ }^{1}$ Cahill Center for Astronomy and Astrophysics, California Institute of Technology, 1200 E California Blvd, Pasadena, CA 91125, USA \\ ${ }^{2}$ McGill University, Department of Physics \& McGill Space Institute, 3600 Rue University, Montréal, QC H3A 2T8, Canada \\ ${ }^{3}$ Department of Astronomy and Astrophysics, University of California, Santa Cruz, 1156 High Street, Santa Cruz, CA 95064, USA \\ ${ }^{4}$ Department of Physics and Astronomy, University of California, Los Angeles, CA 90024, USA
}

Accepted 2021 September 15. Received 2021 September 5; in original form 2021 July 19

\begin{abstract}
The cosmic near-infrared background (NIRB) offers a powerful integral probe of radiative processes at different cosmic epochs, including the pre-reionization era when metal-free, Population III (Pop III) stars first formed. While the radiation from metalenriched, Population II (Pop II) stars likely dominates the contribution to the observed NIRB from the reionization era, Pop III stars - if formed efficiently - might leave characteristic imprints on the NIRB, thanks to their strong Ly $\alpha$ emission. Using a physically motivated model of first star formation, we provide an analysis of the NIRB mean spectrum and anisotropy contributed by stellar populations at $z>5$. We find that in circumstances where massive Pop III stars persistently form in molecular cooling haloes at a rate of a few times $10^{-3} \mathrm{M}_{\odot} \mathrm{yr}^{-1}$, before being suppressed towards the epoch of reionization (EoR) by the accumulated Lyman-Werner background, a unique spectral signature shows up redward of $1 \mu \mathrm{m}$ in the observed NIRB spectrum sourced by galaxies at $z>5$. While the detailed shape and amplitude of the spectral signature depend on various factors including the star formation histories, initial mass function, LyC escape fraction and so forth, the most interesting scenarios with efficient Pop III star formation are within the reach of forthcoming facilities, such as the Spectro-Photometer for the History of the Universe, Epoch of Reionization, and Ices Explorer. As a result, new constraints on the abundance and formation history of Pop III stars at high redshifts will be available through precise measurements of the NIRB in the next few years.
\end{abstract}

Key words: stars: Population II-stars: Population III-galaxies: high-redshift-dark ages, reionization, first stars-diffuse radiation - infrared: diffuse background.

\section{INTRODUCTION}

Population III (Pop III) stars are believed to form in primordial, metal-free gas clouds cooled via molecular hydrogen $\left(\mathrm{H}_{2}\right)$ at very high redshift, well before metal-poor, Population II (Pop II) stars typical for distant galaxies started to form. These first generation of stars at the so-called cosmic dawn were responsible for the onset of cosmic metal enrichment and reionization, and their supernova remnants may be the birthplaces of supermassive black holes observed today (see recent reviews by Bromm 2013; Inayoshi, Visbal \& Haiman 2020). Despite their importance in understanding the cosmic history of star formation, Pop III stars are incredibly difficult to directly detect, even for the upcoming generation of telescopes like the James Webb Space Telescope (JWST) as discussed in Rydberg et al. (2013) and Schauer, Drory \& Bromm (2020), and thus constraints on their properties remain elusive. Nevertheless, the formation and physical properties of Pop III stars have been investigated in detail with theoretical models over the past few decades, and several promising observing methods have been proposed to discover them in the near future.

^E-mail: gsun@astro.caltech.edu
Theoretical models of Pop III stars come in many forms, including simple analytical arguments (e.g. McKee \& Tan 2008), detailed numerical simulations (e.g. Abel, Bryan \& Norman 2002; O'Shea \& Norman 2007; Wise \& Abel 2007; Maio et al. 2010; Greif et al. 2011; Safranek-Shrader et al. 2012; Stacy, Greif \& Bromm 2012; Xu et al. 2016a), and semi-analytic models that balance computational efficiency and physical accuracy (e.g. Trenti \& Stiavelli 2009; Trenti, Stiavelli \& Shull 2009; Crosby et al. 2013; Jaacks et al. 2018; Mebane, Mirocha \& Furlanetto 2018; Visbal, Haiman \& Bryan 2018; Liu \& Bromm 2020) These theoretical efforts reveal a detailed, though still incomplete, picture of how the transition from Pop III to metal-enriched, Pop II star formation might have occurred. Minihaloes above the Jeans/filtering mass scale set by some critical fraction of $\mathrm{H}_{2}$ (Tegmark et al. 1997) and below the limit of atomic hydrogen cooling are thought to host the majority of Pop III star formation since $z \gtrsim 30$, where the rotational and vibrational transitions of collisionally excited $\mathrm{H}_{2}$ dominate the cooling of primordial gas. ${ }^{1}$ The lack of efficient cooling channels yields a Jeans mass of

\footnotetext{
${ }^{1}$ Stars formed out of primordial gas in these molecular cooled haloes are sometimes referred to as Pop III.1 stars, whereas stars formed in atomic cooling haloes that are primordial but affected by previously generated stellar radiation are referred to as Pop III.2 stars.
} 
the star-forming region as high as a few hundred $M_{\odot}$, producing very massive and isolated Pop III stars in the classical picture (Bromm \& Larson 2004). However, simulations indicate that even modest initial angular momentum of the gas in minihaloes could lead to fragmentation of the protostellar core and form Pop III binaries or even multiple systems (e.g. Turk, Abel \& O'Shea 2009; Stacy, Greif $\&$ Bromm 2010; Sugimura et al. 2020), which further complicates the Pop III initial mass function (IMF). Several physical processes contribute to the transition to Pop II star formation. The feedback effect of the Lyman-Werner (LW) radiation background built up by the stars formed is arguably consequential for the formation of Pop III stars. LW photons $(11.2 \mathrm{eV}<h v<13.6 \mathrm{eV})$ can regulate Pop III star formation by photo-dissociating $\mathrm{H}_{2}$ through the two-step Solomon process (Stecher \& Williams 1967) and thereby setting the minimum mass of minihaloes above which Pop III stars can form (Haiman, Rees \& Loeb 1997; Wolcott-Green, Haiman \& Bryan 2011; Holzbauer \& Furlanetto 2012; Stacy et al. 2012; Visbal et al. 2014; Mebane et al. 2018), although some recent studies suggest that $\mathrm{H}_{2}$ self-shielding might greatly alleviate the impact of the LW background (see e.g. Skinner \& Wise 2020). Other important factors to be considered in modelling the transition include the efficiency of metal enrichment (i.e. chemical feedback) from Pop III supernovae (Pallottini et al. 2014; Sarmento, Scannapieco \& Cohen 2018), the Xray background sourced by Pop III binaries that might replenish $\mathrm{H}_{2}$ by catalyzing its formation (Haiman, Abel \& Rees 2000; Hummel et al. 2015; Ricotti 2016), and the residual streaming velocity between dark matter and gas (Tseliakhovich \& Hirata 2010; Fialkov et al. 2012; Naoz, Yoshida \& Gnedin 2012; Schauer et al. 2021). In spite of all the theoretical efforts, substantial uncertainties remain in how long and to what extent Pop III stars might have coexisted with their metal-enriched descendants, leaving the timing and duration of the Pop III to Pop II transition largely unconstrained.

Direct constraints on Pop III stars would be made possible by detecting their emission features. One such feature is the $\mathrm{He} I I \lambda 1640$ line, which is a strong, narrow emission line indicative of a very hard ionizing spectrum typical for Pop III stars (Schaerer 2003). The association of the He I I 1640 line with Pop III stars has been pursued in the context of both targeted observations (e.g. Nagao et al. 2005; Cai et al. 2011; Mas-Ribas, Dijkstra \& Forero-Romero 2016) and statistical measurements via the line-intensity mapping technique (e.g. Visbal, Haiman \& Bryan 2015). While possible identifications have been made for objects such as 'CR7' (Sobral et al. 2015), the measurements are controversial and a solid He II 1640 detection of Pop III stars may not be possible until the operation of nextgeneration ground-based telescopes, such as the E-ELT (Grisdale et al. 2021). A number of alternative (and often complementary) probes of Pop III stars have therefore been proposed, including long gamma-ray bursts associated with the explosive death of massive Pop III stars (Mészáros \& Rees 2010; Toma, Sakamoto \& Mészáros 2011), caustic transits behind lensing clusters (Windhorst et al. 2018), the cosmic near-infrared background (NIRB; Santos, Bromm \& Kamionkowski 2002; Kashlinsky et al. 2004; Fernandez \& Zaroubi 2013; Yang, Wang \& Dai 2015; Helgason et al. 2016; Kashlinsky et al. 2018), spectral signatures in the global 21-cm signal (Thomas \& Zaroubi 2008; Fialkov et al. 2014; Mirocha et al. 2018; Mebane, Mirocha \& Furlanetto 2020), and 21-cm power spectrum (Fialkov et al. 2013, 2014; Qin et al. 2021).

Pop III stars have been proposed as a potential explanation for the observed excess in the NIRB fluctuations (Salvaterra \& Ferrara 2003; Kashlinsky et al. 2004, 2005), which cannot be explained by the known galaxy populations with sensible faint-end extrapolation (Helgason, Ricotti \& Kashlinsky 2012), and their accreting remnants provide a viable explanation for the coherence between the NIRB and the soft cosmic X-ray background detected at high significance (Cappelluti et al. 2013). However, subsequent studies indicate that, for Pop III stars to source a considerable fraction of the observed NIRB, their formation and ionizing efficiencies would need to be so extreme that constraints on reionization and the X-ray background are likely violated (e.g. Madau \& Silk 2005; Helgason et al. 2016). Consequently, some alternative explanations have been proposed, such as the intrahalo light (IHL) radiated by stars stripped away from parent galaxies during mergers (Cooray et al. 2012a; Zemcov et al. 2014), with a major contribution from sources at $z<2$, and accreting direct collapsed black holes that could emit a significant amount of rest-frame, optical-UV emission at $z \gtrsim 12$ due to the absorption of ionizing radiation by the massive accreting envelope surrounding them (Yue et al. 2013b).

Pop III stars alone are likely insufficient to fully explain the source-subtracted NIRB fluctuations observed and separating their contribution to the NIRB from other sources, including Pop II stars that likely co-existed with Pop III stars over a long period of time, will be challenging. Nevertheless, there is continued interest in understanding and modelling potential signatures of Pop III stars in the NIRB (e.g. Kashlinsky et al. 2004, 2005; Yang et al. 2015; Helgason et al. 2016), which is one of only a few promising probes of Pop III in the near term. In particular, Fernandez and Zaroubi (2013, hereafter FZ13) point out that strong Ly $\alpha$ emission from Pop III stars can lead to a 'bump' in the mean spectrum of the NIRB, a spectral signature that can reveal information about physical properties of Pop III stars and the timing of the Pop III to Pop II transition. The soon-to-be-launched satellite Spectro-Photometer for the History of the Universe, Epoch of Reionization, and Ices Explorer (SPHEREx; Doré et al. 2014) has the raw sensitivity to detect the contribution of galaxies during the epoch of reionization (EoR) to the NIRB at high significance (Feng et al. 2019), making it possible, at least in principle, to detect or rule out such spectral features. However, despite significant differences in detailed predictions, previous modelling efforts (e.g. Fernandez \& Komatsu 2006; Cooray et al. 2012b; Yue et al. 2013a; Helgason et al. 2016) have suggested that first galaxies during and before the EoR may only contribute to approximately less than 1 per cent of both the source-subtracted NIRB mean intensity and its angular fluctuations, as measured from a series of deep imaging surveys (e.g. Kashlinsky et al. 2012; Zemcov et al. 2014; Seo et al. 2015). A challenging measurement notwithstanding, unprecedented NIRB sensitivities of space missions like SPHEREx and the Cosmic Dawn Intensity Mapper (CDIM; Cooray et al. 2019) urge the need for an improved modelling framework to learn about the first galaxies from future NIRB measurements.

In this work, we establish a suite of NIRB predictions that are anchored to the latest constraints on the high- $z$ galaxy population drawn from many successful Hubble Space Telescope (HST) programs, such as the Hubble Ultra Deep Field (Beckwith et al. 2006), CANDELS (Grogin et al. 2011), and Hubble Frontier Fields (Lotz et al. 2017). We employ a semi-empirical model to describe the known galaxy population, and then add in a physically motivated, but flexible, model for Pop III stars that allow us to explore a wide range of plausible scenarios. This, in various aspects, improves over previous models, which, e.g. parametrized the fraction of cosmic star formation in Pop III haloes as a function of redshift only and/or employed simpler Pop II models calibrated to earlier data sets (e.g. Cooray et al. 2012b, FZ13; Helgason et al. 2016; Feng et al. 2019). These advancements not only allow more accurate modelling of the contribution to the NIRB from high- $z$ galaxies, but also provide a convenient physical framework to analyse and interpret data sets of 
forthcoming NIRB surveys aiming to quantify the signal level of galaxies during and before reionization.

This paper is organized as follows. In Section 2, we describe how we model the spatial and spectral properties of the NIRB associated with high- $z$ galaxies, using a simple, analytical framework of Pop II and Pop III star formation in galaxies at $z>5$. We present our main results in Section 3, including the predicted NIRB signals, potential spectral imprints due to Pop III star formation, and sensitivity estimates for detecting Pop II and Pop III signals in future NIRB surveys. In Section 4, we show implications for other observables of high- $z$, galaxies that can be potentially drawn from NIRB observations. We discuss a few important caveats and limitations of our results in Section 5, before briefly concluding in Section 6. Throughout this paper, we assume a flat, $\Lambda \mathrm{CDM}$ cosmology consistent with the results from the Planck Collaboration (2016).

\section{MODELS}

\subsection{Star formation history of high-redshift galaxies}

\subsubsection{The formation of Pop II stars}

Following Mirocha, Furlanetto \& Sun (2017), we model the star formation rate density (SFRD) of normal, high- $z$ galaxies as an integral of the star formation rate (SFR) per halo $\dot{M}_{*}\left(M_{h}\right)$ over the halo mass function $n\left(M_{h}\right.$; see also Sun \& Furlanetto 2016; Furlanetto et al. 2017)

$$
\begin{aligned}
\dot{\rho}_{*}^{\mathrm{II}}(z) & =\int_{M_{h, \text { min }}^{\mathrm{II}}} n\left(M_{h}\right) \dot{M}_{*}\left(M_{h}, z\right) d M_{h} \\
& =\int_{M_{h, \text { min }}^{\mathrm{II}}} n\left(M_{h}\right) f_{*}\left(M_{h}, z\right) \frac{\Omega_{b}}{\Omega_{m}} \dot{M}_{h}\left(M_{h}, z\right) d M_{h},
\end{aligned}
$$

where $M_{h, \text { min }}^{\mathrm{II}}$ is generally evaluated at a virial temperature of $T_{\text {vir }}=10^{4} \mathrm{~K}$, a free parameter in our model above which Pop II are expected to form due to efficient cooling via neutral atomic lines (Oh \& Haiman 2002), namely $M_{h, \text { min }}^{\mathrm{II}}=M_{h, \text { max }}^{\mathrm{III}} \cdot \dot{M}_{*}\left(M_{h}\right)$ is further specified by a star formation efficiency (SFE), $f_{*}$, defined to be the fraction of accreted baryons that eventually turn into stars, and the mass growth rate, $\dot{M}_{h}$, of the dark matter halo. We exploit the abundance matching technique to determine the mean halo growth histories by matching halo mass functions at different redshifts. As illustrated in Furlanetto et al. (2017) and Mirocha, La Plante \& Liu (2021), the abundance-matched accretion rates given by this approach are generally in good consistency with results based on numerical simulations (Trac, Cen \& Mansfield 2015) for atomic cooling haloes at $5 \lesssim z \lesssim 10$ (but see Schneider, Giri \& Mirocha 2021 for a comparison with estimates based on the extended Press-Schechter formalism). Even though effects like mergers and the stochasticity in $\dot{M}_{h}$ introduce systematic biases between the inferences made based on merger trees and abundance matching, such biases can be largely eliminated by properly normalizing the nuisance parameters in the model (Mirocha et al. 2021). By calibrating to the latest observational constraints on the galaxy UV luminosity function (UVLF), Mirocha et al. (2017) estimate $f_{*}$ to follow a double power law in halo mass (the dpl model)

$f_{*}^{\mathrm{dpl}}\left(M_{h}\right)=\frac{f_{*, 0}}{\left(\frac{M_{h}}{M_{\mathrm{p}}}\right)^{\gamma / \mathrm{lo}}+\left(\frac{M_{h}}{M_{\mathrm{p}}}\right)^{\gamma / \mathrm{hi}}}$,

with no evident redshift evolution, in agreement with other recent work (e.g. Mason, Trenti \& Treu 2015; Tacchella et al. 2018;
Behroozi et al. 2019; Stefanon et al. 2021). The evolution of $f_{*}$ for low-mass haloes is however poorly constrained by the faint-end slope of the UVLF, and can be highly dependent on the regulation of feedback processes (Furlanetto et al. 2017; Furlanetto 2021) and the burstiness of star formation (Furlanetto \& Mirocha 2021). Therefore, in addition to the baseline dpl model, we consider two alternative parametrization - one suggested by Okamoto, Gao \& Theuns (2008) that allows a steep $\operatorname{drop}$ of $f_{*}$ for low-mass haloes (the steep model)

$f_{*}^{\text {steep }}\left(M_{h}\right)=\left[1+\left(2^{\mu / 3}-1\right)\left(\frac{M_{h}}{M_{\text {crit }}}\right)^{-\mu}\right]^{-3 / \mu}$,

and the other that imposes a constant floor on the SFE of 0.005 (the floor model). In this work, we take the same best-fitting parameters as those given by Mirocha et al. (2017) to define the two reference Pop II models, namely $f_{*, 0}=0.05, M_{\mathrm{p}}=2.8 \times 10^{11}, \gamma_{\mathrm{lo}}=0.49, \gamma_{\mathrm{hi}}$ $=-0.61$, with $\mu=1$ and $M_{\text {crit }}=10^{10} M_{\odot}$ for the steep model. ${ }^{2}$ With the three variants of our Pop II SFE model, we aim to bracket a reasonable range of possible low mass/faint-end behaviour, and emphasize that future observations by the JWST (e.g. Furlanetto et al. 2017; Yung et al. 2019) and line-intensity mapping surveys (e.g. Park et al. 2020; Sun et al. 2021) can place tight constraints on these models.

\subsubsection{The formation of Pop III stars}

While the star formation history of Pop II stars may be reasonably inferred by combing existing observational constraints up to $z \sim 10$ with physically motivated extrapolations towards higher redshifts, the history of Pop III stars is only loosely constrained by observations. Several recent studies (e.g. Visbal et al. 2014; Jaacks et al. 2018; Mebane et al. 2018; Sarmento et al. 2018; Liu \& Bromm 2020) investigate the formation of Pop III stars under the influence of a variety of feedback processes, including the LW background and supernovae. In general, these models find that Pop III SFRD increases steadily for approximately $200 \mathrm{Myr}$ since the onset of Pop III star formation at $z \gtrsim 30$, before sufficiently strong feedback effects can be established to regulate their formation. In detail, however, the predicted Pop III SFRDs differ substantially in both shape and amplitude. Massive Pop III star formation can persist in minihaloes for different amounts of time depending on factors such as the strength of LW background and the efficiency of metal enrichment (which, in turn, depends on how metals can be produced, retained and mixed within minihaloes). Consequently, the formation of Pop III stars can either terminate as early as $z>$ 10 in some models, or remain a non-negligible rate greater than $10^{-4} \mathrm{M}_{\odot} \mathrm{yr}^{-1} \mathrm{Mpc}^{-3}$ through the post-reionization era in others. Given the large uncertainty associated with the Pop III SFRD, we follow Mirocha et al. (2018) and account for the Pop III to Pop II transition with a simple descriptive model, which offers a flexible way to simultaneously capture the physics of Pop III star formation and encompass a wide range of possible scenarios. We defer the interested readers to that paper and only provide a brief summary here.

We assume that Pop III stars can only form in minihaloes with halo mass between $M_{h, \text { min }}^{\mathrm{III}}$ and $M_{h, \max }^{\mathrm{III}}$ at a constant rate $\dot{M}_{*}^{\mathrm{III}}$ per halo, in

\footnotetext{
${ }^{2}$ The SFE parameters taken are fit to the observed UVLFs measured by Bouwens et al. (2015) at $6<z<8$, which agree reasonably well with the most recent measurements in (e.g. Bouwens et al. 2021).
} 
which case the Pop III SFRD can be written as

$\dot{\rho}_{*}^{\mathrm{III}}(z)=\dot{M}_{*}^{\mathrm{III}} \int_{M_{h, \text { min }}^{\mathrm{III}}}^{M_{h, \max }^{\mathrm{III}}} n\left(M_{h}\right) d M_{h}$.

The minimum mass, $M_{h \text {, } \operatorname{III}}^{\mathrm{III}}$, of Pop III star-forming haloes is set by the threshold for effective $\mathrm{H}_{2}$ cooling, regulated in response to the growing LW background following Visbal et al. (2014). The maximum mass, $M_{h, \text { max }}^{\mathrm{III}}$, of Pop III star-forming haloes is controlled by two free parameters, which set the critical amount of time individual haloes spend in the Pop III phase, $\mathcal{T}_{c}$, as well as a critical binding energy, $\mathcal{E}_{c}$, at which point haloes are assumed to transition from Pop III to Pop II star formation. The first condition effectively results in a fixed amount of stars (and metals) produced per halo in our model, and thus serves as a limiting case in which the Pop III to Pop II transition is governed by the production of metals. The second condition enforced by $\mathcal{E}_{c}$ provides a contrasting limiting case, in which the transition from Pop III to Pop II is instead governed by metal retention. In practice, $\mathcal{E}_{c}$ may range from as small as the typical energy output of a supernova $\left(\sim 10^{51} \mathrm{erg}\right)$ to a few hundred times larger. ${ }^{3}$ It is worth noting that, rather than quantifying the impact of metal enrichment on Pop III star formation and the corresponding NIRB signal through a global volume-filling factor of metal-enriched intergalactic medium (IGM) due to galactic outflows (see e.g. Yang et al. 2015), we use $\mathcal{T}_{c}$, and $\mathcal{E}_{c}$ to control the Pop III to Pop II transition. Although this approach does not invoke the metallicity of haloes explicitly, it is flexible enough to produce SFRDs that are in good agreement with more sophisticated models, which do link the Pop III to Pop II transition to halo metallicity (e.g. Mebane et al. 2018). Finally, for simplicity, we assume blackbody spectrum for Pop III stars and scale the ionizing flux with the parameter $Q(\mathrm{H})$, which we describe in more detail in Section 2.2.2.

Fig. 1 shows the star formation histories of Pop II and Pop III stars calculated from a collection of models we consider in this work. Values of key model parameters adopted are summarized in Table 1. Specifically, three different cases (all permitted by current observational constraints, see e.g. Mirocha et al. 2017) of extrapolating Pop II star formation down to low-mass, atomic-cooling haloes unconstrained by the observed UVLFs are referred to as Model I (dpl, see equation 2), Model II (steep, see equation 3), and Model III (floor), respectively. $f_{\text {esc }}$ and $f_{\text {esc, LW }}$ represent the escape fractions of Lyman continuum (LyC) and LW photons, respectively. Four Pop III models with distinct SFRDs resulting from different combinations of $\dot{M}_{*}^{\mathrm{III}}, \mathcal{T}_{c}$, and $\mathcal{E}_{c}$ are considered. Model A represents an optimistic case with extremely efficient formation of massive, Pop III stars that leads to a prominent signature on the NIRB. To form $100 \mathrm{M}_{\odot}$ Pop III stars that yields $Q(\mathrm{H}) \sim 10^{50} \mathrm{~s}^{-1}$ at a rate as high as $\dot{M}_{*}^{\mathrm{III}} \sim 10^{-3} \mathrm{M}_{\odot} \mathrm{yr}^{-1}$ in minihaloes with a typical baryonic mass accretion rate of $10^{-3}-10^{-2} \mathrm{M}_{\odot} \mathrm{yr}^{-1}$ (e.g. Greif et al. 2011; Susa, Hasegawa \& Tominaga 2014), the SFE must be exceedingly high and even close to unity over long time-scales. This, in turn, requires a relatively inefficient coupling between the growth of Pop III stars and the radiative and mechanical feedback. Models B, C, and D are our model approximations to Pop III histories derived with the semi-analytical approach described in Mebane et al. (2018). Similar to Model A, all these models yield Pop III SFRDs regulated by LW feedback associated with Pop II and/or Pop III stars themselves, as controlled by the parameters $f_{\mathrm{esc}, \mathrm{LW}}^{\mathrm{II}}$ and $f_{\mathrm{esc}, \mathrm{LW}}^{\mathrm{III}}$. We note that

\footnotetext{
${ }^{3}$ As discussed in Mirocha et al. (2018), it is likely that $\dot{M}_{*}^{\text {III }}, \mathcal{T}_{c}$, and $\mathcal{E}_{c}$ are actually positively correlated with each other in reality, but we ignore such subtleties here to maximally explore the possible scenarios.
}

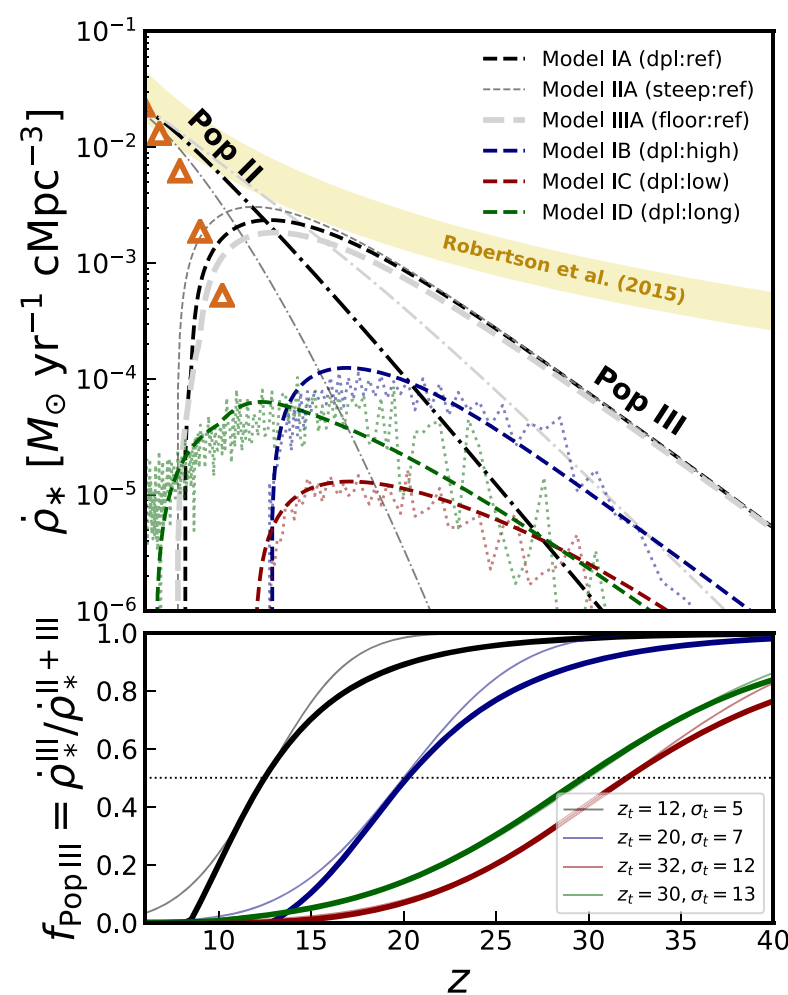

Figure 1. Pop II and Pop III star formation histories in different models considered in this work, as specified in Table 1. Top: SFRDs of Pop II (dashdotted) and Pop III (dashed) stars. The black curves represent our reference model (Model IA), with the thin dark grey curve and the thick light grey curve representing variations where the Pop II SFE follows the steep (Model II) and floor (Model III) models, respectively. The bottom set of three dotted curves show the Pop III histories derived with the semi-analytical approach in Mebane et al. (2018), to which Models IB, IC, and ID are calibrated. The shaded region and open triangles represent the cosmic SFRD inferred from the maximum-likelihood model by Robertson et al. (2015) and the observed SFRD (integrated to a limiting SFR of $0.3 \mathrm{M}_{\odot} \mathrm{yr}^{-1}$ ) up to $z=10$ determined by Oesch et al. (2018), respectively. Bottom: the stellar population transition represented by the ratio of Pop III and total SFRDs. For comparison, approximations made with the functional form $f_{\text {Pop III }}(z)=1 / 2+\operatorname{erf}[(z-$ $\left.\left.z_{t}\right) / \sigma_{t}\right] / 2$ are shown by the thin curves.

setting $f_{\text {esc.LW }}^{\text {III }}$ to zero (as in Model C) is only meant to turn the LW feedback off, since in reality the escape fraction of LW photons tends to be order of unity in the far-field limit (see e.g. Schauer et al. 2017). Besides the LW feedback that sets the end of the Pop III era, the amplitude of the Pop III SFRD is also determined by the prescription of Pop III star formation. Among the three models, Model C approximates the scenario where Pop III stars with a normal IMF form at a low level of stellar mass produced per burst, which yields NIRB signals likely inaccessible to upcoming observations, whereas Models B and D approximate scenarios where Pop III stars form more efficiently and persistently, respectively, and if massive enough $\left(M_{*} \sim 500 \mathrm{M}_{\odot}\right)$, can leave discernible imprints on the NIRB. For comparison, two additional cosmic SFRDs are shown: (i) that inferred from Robertson et al. (2015) by integrating the UVLFs down to $L_{\mathrm{UV}} \sim 0.001 L_{*}$ (yellow band), and (ii) that reported in Oesch et al. (2018) which includes observed galaxies with $\dot{M}_{*} \gtrsim 0.3 \mathrm{M}_{\odot} \mathrm{yr}^{-1}$ (open triangles).

To put things into the context of the literature, we show in the lower panel of Fig. 1 the fraction of stars that are Pop III at each redshift. Predictions from our models are shown together with 
Table 1. Parameter values in the reference models of Pop II and Pop III star formation.

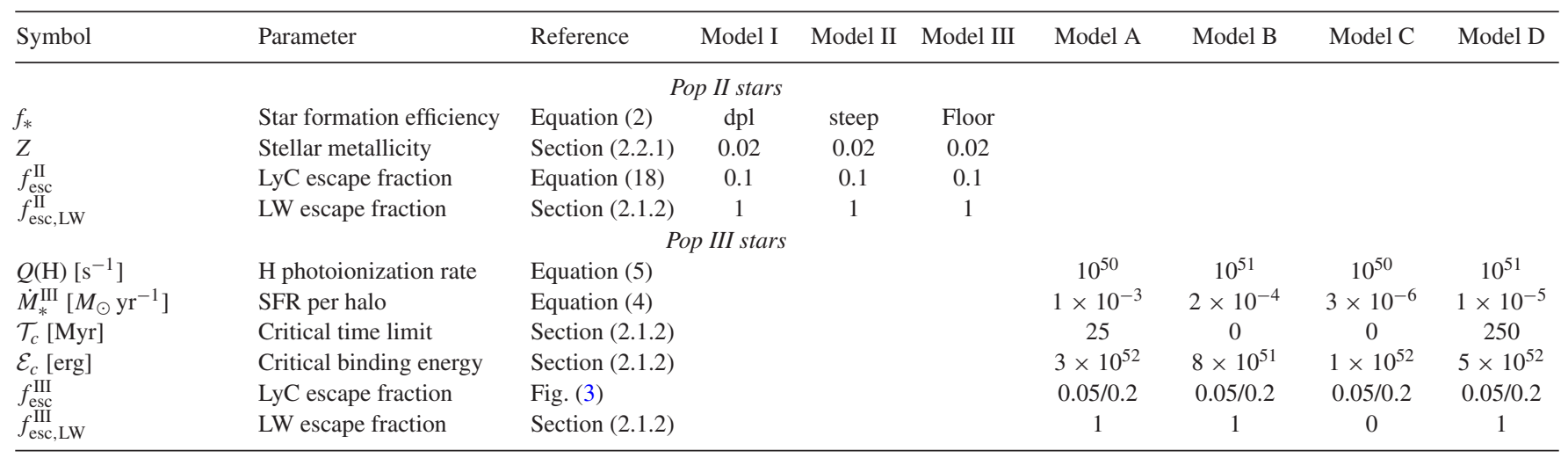

approximations made using the functional form $f_{\text {Pop III }}(z)=1 / 2+$ $\operatorname{erf}\left[\left(z-z_{t}\right) / \sigma_{t}\right] / 2$, which is frequently adopted in the literature to estimate the Pop III contribution (e.g. Cooray et al. 2012b; Fernandez $\&$ Zaroubi 2013; Feng et al. 2019). It can be seen that, compared with the phenomenological description using the error function, our physical models imply a more extended early phase with the Pop II SFRD gradually catching up. The late-time behaviour is characterized by how sharply the Pop III phase terminates, which in turn depends on whether $\mathcal{T}_{c}$ or $\mathcal{E}_{c}$ is in operation.

\subsection{Spectra of high- $z$ galaxies}

In this section, we introduce our approach to modelling the spectral energy distribution (SED) of high- $z$ galaxies. An illustrative example is shown first in Fig. 2, which includes Pop II and Pop III spectra, with and without the additional contribution from nebular emission. Each component of the SED is described in more detail in Sections 2.2.12.2.4. We note that for the NIRB contribution from nebular line emission we only include hydrogen lines like Ly $\alpha$, the strongest emission line from high- $z$ galaxies in the near-infrared, even though lines such as the He II $\lambda 1640$ line (for Pop III stars) could also be interesting - in the sense of both their contributions to the NIRB and their spatial fluctuations that can be studied in the lineintensity mapping regime. In the following subsections, we specify the individual components of the NIRB according to how they are implemented in ARES $^{4}$ (Mirocha 2014), which was used to conduct all the calculations in this work.

\subsubsection{Direct stellar emission}

The direct stellar emission from the surfaces of Pop II and Pop III stars is the foundation upon which the full SED of high- $z$ galaxies is built in our models. It depends in general on the stellar IMF, metallicity, and assumed star formation history of galaxies. For the SED of Pop II stars, we adopt the single-star models calculated with the stellar population synthesis (SPS) code BPASS v1.0 (Eldridge \& Stanway 2009), which assume a Chabrier IMF (Chabrier 2003) and a metallicity of $Z=0.02^{5}$ in the default case. As is common in many semi-empirical models, we further assume a constant star formation

\footnotetext{
${ }^{4}$ https://github.com/mirochaj/ares

${ }^{5}$ While it is plausible to assume sub-solar metallicity for galaxies during and before reionization given the rate of metal enrichment expected (Furlanetto et al. 2017), the exact value of $Z$ is highly uncertain and lowering it by 1 or 2 dex does not change our results qualitatively.
}

history, for which the rest-UV spectrum evolves little after $\sim 100 \mathrm{Myr}$. We therefore adopt $100 \mathrm{Myr}$ as the fiducial stellar population age, as in Mirocha et al. $(2017,2018)$, which is a reasonable assumption for high- $z$ galaxies with high specific SFRs of the order $10 \mathrm{Gyr}^{-1}$ (e.g. Stark et al. 2013). For Pop III stars, the SED is assumed to be a $10^{5} \mathrm{~K}$ blackbody for simplicity, which is appropriate for stars with masses $\gtrsim 100 M_{\odot}$ (e.g. Tumlinson \& Shull 2000; Schaerer 2002). We further assume that Pop III stars form in isolation, one after the next, which results in a time-independent SED.

\subsubsection{Lya emission}

The full spectrum of a galaxy must also account for reprocessed emission originating in galactic $\mathrm{H}$ II regions. The strongest emission line is $\operatorname{Ly} \alpha$ - because $\operatorname{Ly} \alpha$ emission is mostly due to the recombination of ionized hydrogen, a simple model for its line luminosity can be derived assuming ionization equilibrium and case-B recombination. Specifically, the photoionization equilibrium is described by defining a volume $V_{\mathrm{S}}$ within which the ionization rate equals the rate of recombination

$\alpha_{\mathrm{B}} n_{e}^{\text {neb }} n_{\mathrm{HII}}^{\mathrm{neb}} V_{\mathrm{S}}=Q(\mathrm{H})$,

where $\alpha_{\mathrm{B}}=\alpha_{2^{2} \mathrm{P}}^{\text {eff }}+\alpha_{2^{2} \mathrm{~S}}^{\text {eff }}$ is the total case-B recombination coefficient as the sum of effective recombination coefficients to the $2^{2} \mathrm{P}$ and $2^{2} \mathrm{~S}$ states, and $Q(\mathrm{H})$ is the photoionization rate in $\mathrm{s}^{-1}$. It is important to note that, in previous models of the NIRB, an additional factor $\left(1-f_{\text {esc }}\right)$ is often multiplied to $Q_{\mathrm{H}}$. It is intended to roughly account for the fraction of ionizing photons actually leaking into the IGM, and therefore not contributing to the absorption and recombination processes that source the nebular emission. We have chosen not to take this simple approximation in our model, but to physically connect $f_{\text {esc }}$ with the profile of ionizing radiation instead (see Section 2.4). The Ly $\alpha$ emission $\left(2^{2} \mathrm{P} \rightarrow 1^{2} \mathrm{~S}\right)$ is associated with the recombination of ionized hydrogen to the $2^{2} \mathrm{P}$ state, so its line luminosity can be written as

$l^{\mathrm{Ly} \alpha}=h v_{\mathrm{Ly} \alpha} \alpha_{2^{2} \mathrm{P}}^{\mathrm{eff}} n_{e}^{\mathrm{neb}} n_{\mathrm{HII}}^{\mathrm{neb}} V_{\mathrm{S}}=\frac{Q(\mathrm{H}) h v_{\mathrm{Ly} \alpha} \alpha_{2^{2} \mathrm{P}}^{\mathrm{eff}}}{\alpha_{\mathrm{B}}}$,

or in the volume emissivity $\epsilon_{v}^{\text {Ly } \alpha}$

$\epsilon_{v}^{\mathrm{Ly} \alpha} V_{\mathrm{S}}=Q(\mathrm{H}) f_{\mathrm{Ly} \alpha} h v_{\mathrm{Ly} \alpha} \phi\left(v-v_{\mathrm{Ly} \alpha}\right)$,

where $f_{\mathrm{Ly} \alpha}=\alpha_{2^{2} \mathrm{P}}^{\text {eff }} / \alpha_{\mathrm{B}} \approx 2 / 3$ is the fraction of recombinations ending up as Ly $\alpha$ radiation and $\phi\left(v-v_{\mathrm{Ly} \alpha}\right)$ is the line profile, which we assume to be a delta function in our model. 


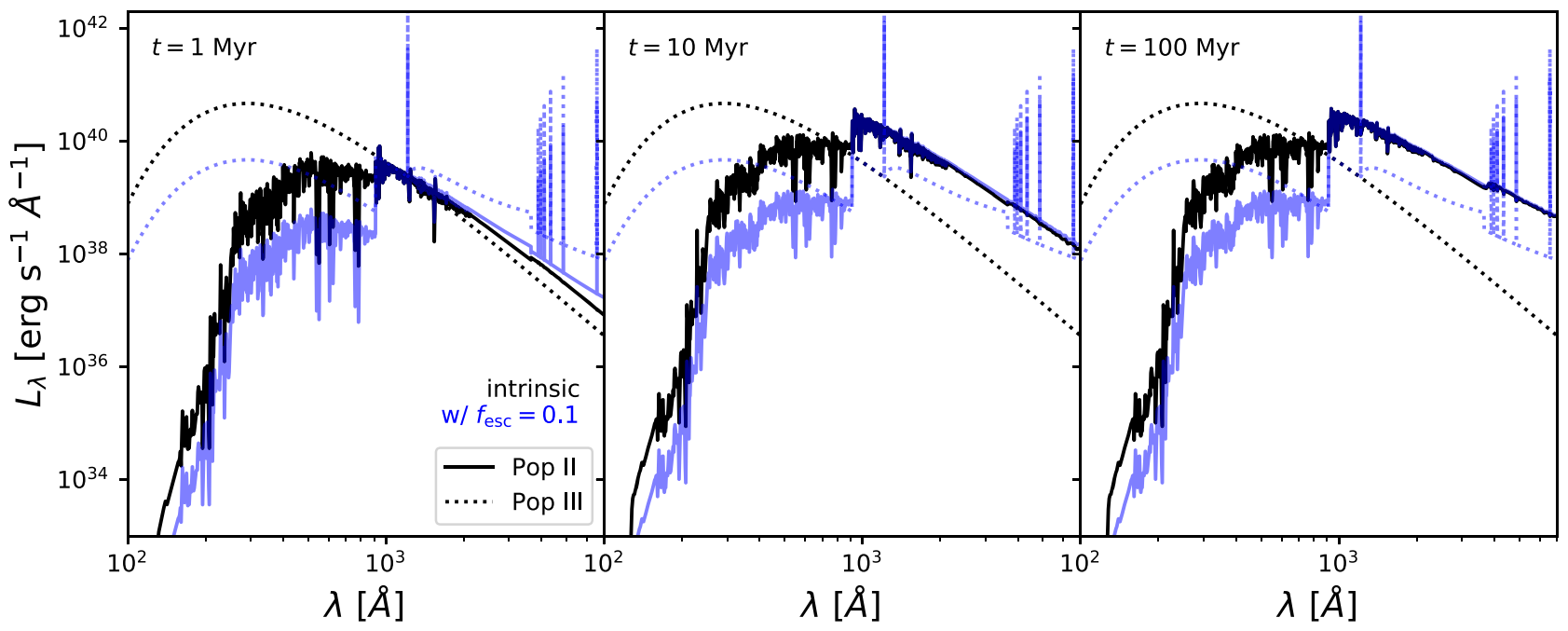

Figure 2. Example spectra of stellar populations employed in this work. In each panel, black curves show the intrinsic Pop II (solid) and Pop III (dotted) stellar continuum. For Pop II, we show models that assume a constant SFR of $1 \mathrm{M}_{\odot} \mathrm{yr}^{-1}$ with ages of 1, 10, and $100 \mathrm{Myr}$ (left to right). Pop III models are the same in each panel, and assume a single star with ionizing luminosity of $10^{48}$ photons s ${ }^{-1}$. Blue lines show the nebular continuum and nebular line emission (see Sections 2.2.2-2.2.4), powered by the absorption of Lyman continuum photons assuming an escape fraction of 10 per cent. We adopt the $t=100 \mathrm{Myr}$ models (right-most panel) throughout, a time-scale on which the rest-UV spectrum will asymptote to a constant level. The early-time evolution is included to demonstrate the nebular continuum treatment.

Now, with $\epsilon_{b}$ being the number of ionizing photons emitted per stellar baryon, which we derive from the stellar spectrum generated with BPASS (see Section 2.2.1), we can write

$Q(\mathrm{H}) \approx \epsilon_{b} \dot{\rho}_{*} V_{\mathrm{S}} / m_{p}$,

where $m_{p}$ is the mass of the proton. The volume emissivity of Ly $\alpha$ photons is then

$\bar{\epsilon}_{v}^{\mathrm{Ly} \alpha} \mathrm{d} v=\frac{\dot{\rho}_{*}}{m_{p}} f_{\mathrm{Ly} \alpha} \epsilon_{b} h v_{\mathrm{Ly} \alpha} \phi\left(v-v_{\mathrm{Ly} \alpha}\right) d \nu$.

It is also important to note that the above calculations assume Ly $\alpha$ emission is completely described by the case-B recombination of hydrogen, which only accounts for the photoionization from the ground state. In practice, though, additional effects such as collisional excitation and ionization may cause significant departures from the case-B assumption. These effects have been found to be particularly substantial for metal-free stars, which typically have much harder spectra than metal-enriched stars (see e.g. Raiter, Schaerer \& Fosbury 2010 and Mas-Ribas et al. 2016 for details). Due to the deficit of cooling channels, low-metallicity nebulae can have efficient collisional effects that induce collisional excitation/ionization and ionization from excited levels, ${ }^{6}$ which all lead to a higher Ly $\alpha$ luminosity than expected under the case-B assumption. This enhancement is found to scale with the mean energy of ionizing photons. Meanwhile, density effects can mix $2^{2} \mathrm{~S}$ and $2^{2} \mathrm{P}$ states, thus altering the relative importance of $\operatorname{Ly} \alpha$ and two-photon emission. This is determined simply by $\alpha_{2^{2} \mathrm{P}}^{\text {eff }}$ and $\alpha_{2^{2} \mathrm{~S}}^{\text {eff }}$ in the low-density limit. When density effects are nontrivial as $n_{e}$ becomes comparable to the critical density $n_{e, \text { crit }}$ (at which $2^{2} \mathrm{~S} \rightarrow 2^{2} \mathrm{P}$ transition rate equals the radiative decay rate), collisions may de-populate the $2^{2} \mathrm{~S}$ state of hydrogen before spontaneous decay occurs. In this case, Ly $\alpha$ is further enhanced at the expense of two-photon emission.

For simplicity, in our model, we introduce an ad hoc correction factor $\mathcal{D}_{\mathrm{B}}$ to account for the net boosting effect of Ly $\alpha$ emission from Pop III star-forming galaxies. Throughout our calculations, we use a fiducial value of $\mathcal{D}_{\mathrm{B}}=2$ for Pop III stars, a typical value for very massive Pop III stars considered in this work, and $\mathcal{D}_{\mathrm{B}}=1$ for Pop II stars. The volume emissivity after correcting for case-B departures is then

$\bar{\epsilon}_{v}^{\mathrm{Ly} \alpha} \mathrm{d} v=\frac{\dot{\rho}_{*}(z)}{m_{p}} \epsilon_{b} h v_{\mathrm{Ly} \alpha} \mathcal{D}_{\mathrm{B}} \phi\left(v-v_{\mathrm{Ly} \alpha}\right) \mathrm{d} v$.

We also note that, by default, our nebular line model also includes Balmer series lines, using line intensity values from table 4.2 of Osterbrock \& Ferland (2006).

\subsubsection{Two-photon emission}

For two-photon emission $\left(2^{2} \mathrm{~S} \rightarrow 1^{2} \mathrm{~S}\right)$, the probability of transition producing one photon with frequency in range $\mathrm{d} x=\mathrm{d} \nu / \nu_{\text {Ly } \alpha}$ can be modelled as (Fernandez \& Komatsu 2006)

$P\left(x^{\prime}\right)=1.307-2.627 x^{\prime 2}+2.563 x^{\prime 4}-51.69 x^{\prime 6}$,

where $x^{\prime}=x-0.5$. Note that $P\left(x^{\prime}\right)$ is symmetric around $x=0.5$ as required by energy conservation and is normalized such that $\int_{0}^{1} P(x) \mathrm{d} x=1$. By analogy to Ly $\alpha$ emission, the two-photon volume emissivity under the case-B assumption can be written as

$\bar{\epsilon}_{v}^{2 \gamma} d v=\frac{\dot{\rho}_{*}(z)}{m_{p}}\left(1-f_{\mathrm{Ly} \alpha}\right) \epsilon_{b} \frac{2 h v}{v_{\mathrm{Ly} \alpha}} P\left(v / \nu_{\mathrm{Ly} \alpha}\right) d v$.

${ }^{6}$ Mas-Ribas et al. (2016) find the column density and optical depth of hydrogen atoms in the first excited state to be very small in their photoionization simulations using CLOUDY (Ferland et al. 2013), meaning that the photoionization from $n=2$ is likely inconsequential for the boosting.

\subsubsection{Free-free \& free-bound emission}

The free-free and free-bound (recombination to different $n$ levels of hydrogen) emission also contribute to the nebular continuum. The 
specific luminosity and the volume emissivity are related by

$l_{v}=\frac{\epsilon_{\nu} Q_{\mathrm{H}}}{n_{e} n_{p} \alpha_{\mathrm{B}}}$,

where $\alpha_{\mathrm{B}}$ as a function of gas temperature $T_{\mathrm{g}}$ is given by

$\alpha_{\mathrm{B}}=\frac{2.06 \times 10^{-11}}{T_{\mathrm{g}}^{1 / 2}} \phi_{2}\left(T_{\mathrm{g}}\right) \sim \frac{2.06 \times 10^{-11}}{T_{\mathrm{g}}^{1 / 2}} \mathrm{~cm}^{3} \mathrm{~s}^{-1}$,

where $\phi_{2}\left(T_{\mathrm{g}}\right)$ is a dimensionless function of gas temperature that is of order unity for a typical temperature of $\mathrm{H}$ II regions $T_{\mathrm{g}} \approx 2 \times 10^{4} \mathrm{~K}$. We take the following expression given by Dopita \& Sutherland (2003) for the volume emissivity including both free-free and freebound emission

$\epsilon_{v}^{\text {free }}=4 \pi n_{e} n_{p} \gamma_{\mathrm{c}}(\nu) \frac{e^{-h v / k T_{g}}}{T_{\mathrm{g}}^{1 / 2}} \mathrm{erg} \mathrm{cm}^{-3} \mathrm{~s}^{-1} \mathrm{~Hz}^{-1}$,

where a continuous emission coefficient, $\gamma_{c}(v)$, in units of $\mathrm{cm}^{3} \mathrm{erg} \mathrm{s}^{-1} \mathrm{~Hz}^{-1}$ is introduced to describe the strengths of freefree and free-bound emission. Values of $\gamma_{c}$ as a function of frequency are taken from table 1 of Ferland (1980), which yield a nebular emission spectrum in good agreement with the reprocessed continuum predicted by photoionization simulations. We can then write the emissivity as

$\bar{\epsilon}_{v}^{\text {free }} \mathrm{d} v=\frac{4 \pi}{2.06 \times 10^{-11}} \frac{\dot{\rho}_{*}(z)}{m_{p}} \epsilon_{b} e^{-h v / k T} \gamma_{c}(v) \mathrm{d} v$.

Note that the volume emissivities shown above with an overbar can be considered as the first moment of luminosity, namely averaging the luminosity per halo over the halo mass function

$\bar{\epsilon}_{v}^{i}(z)=\int n\left(M_{h}\right) l_{v}^{i}\left(M_{h}, z\right) d M_{h}$,

where $l_{v}^{i}\left(M_{h}, z\right)$ is the specific luminosity of component $i$ as a function of halo mass and redshift, which can be obtained by simply replacing the SFRD, $\dot{\rho}_{*}$, in equation (16) with the SFR, $\dot{M}_{*}$.

\subsection{Mean NIRB intensity}

For a given source population, the mean intensity at an observed frequency $v_{0}$ of the NIRB can be described by evolving the volume emissivity through cosmic time (i.e. the solution to the cosmological radiative transfer equation)

$J_{v_{0}}(z)=\frac{1}{4 \pi} \int_{z_{0}}^{z} \mathrm{~d} z^{\prime} \frac{\mathrm{d} \ell}{\mathrm{d} z^{\prime}} \frac{\left(1+z_{0}\right)^{3}}{\left(1+z^{\prime}\right)^{3}} \bar{\epsilon}_{v^{\prime}}^{\text {prop }}\left(z^{\prime}\right) e^{-\tau_{\mathrm{HI}}\left(v, z_{0}, z^{\prime}\right)}$,

where $d \ell / d z^{\prime}=c /\left[H\left(z^{\prime}\right)\left(1+z^{\prime}\right)\right]$ is the proper line element and $v^{\prime}$ $=v_{0}\left(1+z^{\prime}\right) /\left(1+z_{0}\right)$. For $z_{0}=0$, the average, comoving volume emissivity is related to the proper volume emissivity by $\bar{\epsilon}_{v}(z)=$ $\bar{\epsilon}_{v}^{\text {prop }}(z) /(1+z)^{3}$. If one assumes the IGM is generally transparent to NIRB photons from high redshifts, then the mean intensity can be simplified to (e.g. Fernandez et al. 2010; Yang et al. 2015)

$J_{v} \equiv \bar{I}_{v}=\frac{c}{4 \pi} \int \mathrm{d} z \frac{\bar{\epsilon}_{v^{\prime}}(z)}{H(z)(1+z)}$,

or the per logarithmic frequency form (e.g. Cooray et al. 2012b),

$v \bar{I}_{v}=\frac{c}{4 \pi} \int \mathrm{d} z \frac{v^{\prime} \bar{\epsilon}_{v^{\prime}}(z)}{H(z)(1+z)^{2}}$.

However, the IGM absorption may not be negligible for certain NIRB components, such as the highly resonant Ly $\alpha$ line, in which case the radiative transfer equation must be solved in detail. To approximate the attenuation by a clumpy distribution of intergalactic H I clouds, we adopt the IGM opacity model from Madau (1995). In
ARES, equation (19) is solved numerically following the algorithm introduced in Haardt \& Madau (1996).

\subsection{NIRB fluctuations}

Using the halo model established by Cooray \& Sheth (2002), we can express the $3 \mathrm{D}$, spherically averaged power spectrum of the NIRB anisotropy associated with high- $z$ galaxies as a sum of three terms

$P_{\mathrm{NIR}}(k, z)=P_{2 \mathrm{~h}}(k, z)+P_{1 \mathrm{~h}}(k, z)+P_{\text {shot }}(z)$,

where each term is composed of direct stellar emission and/or nebular emission. In our model, we divide the emission from a galaxy into two components: (1) a discrete, point-source-like component sourced by direct stellar emission and contributing to the two-halo and shotnoise terms; and (2) a continuous, spatially extended component sourced by nebular emission from the absorption of ionizing photons in the circumgalactic medium (CGM) or IGM by neutral gas and contributing to the two-halo and one-halo terms.

Specifically, the two-halo term is proportional to the power spectrum of the underlying dark matter density field

$P_{2 \mathrm{~h}}(k)=\left[\int n\left(M_{h}\right) b\left(M_{h}\right) \sum_{i} l_{v}^{i}\left(M_{h}\right) u_{i}\left(k \mid M_{h}\right) \mathrm{d} M_{h}\right]^{2} P_{\delta \delta}(k)$,

where the summation is over the stellar and nebular components of galactic emission and $u(k)$ is the normalized Fourier transform of the halo flux profile. $P_{\delta \delta}$ is the dark matter power spectrum obtained from CAMB (Lewis, Challinor \& Lasenby 2000). We take $u_{*}(k)=1$ for the halo luminosity of direct stellar emission $\left(l_{v}^{*}\right)$ and derive the functional form $u_{\mathrm{n}}(k)$ for the halo luminosity of nebular emission $\left(l_{v}^{\mathrm{Ly} \alpha}, l_{v}^{2 \gamma}, l_{v}^{\mathrm{ff}+\mathrm{fb}}\right)$ using the profile of ionizing flux emitted from the galaxy. Because the one-halo term is only sourced by nebular emission, it can be expressed as

$P_{1 \mathrm{~h}}(k)=\int n\left(M_{h}\right)\left[\sum_{j} l_{v}^{j}\left(M_{h}\right) u_{\mathrm{n}}\left(k \mid M_{h}\right)\right]^{2} \mathrm{~d} M_{h}$,

where the summation is over the different types of nebular emission described in Section 2.2.2-2.2.4. Finally, the scale-independent shotnoise term is solely contributed by direct stellar emission, namely

$P_{\text {shot }}=\int n\left(M_{h}\right)\left[l_{v}^{*}\left(M_{h}\right)\right]^{2} \mathrm{~d} M_{h}$.

For simplicity, we ignore the stochasticity in luminosity-halo mass relations for the ensemble of galaxies. Its effect on the shape of $P_{\text {NIR }}(k)$ may be quantified by assuming a probability distribution function (e.g. Sun et al. 2019), but is likely subdominant to (and degenerate with) the systematic uncertainties associated with the relations themselves.

\subsubsection{The radial profile of nebular emission}

We stress that in our model, the nebular emission is assumed to be smooth and thus contributes to $P_{2 \mathrm{~h}}$ and $P_{1 \mathrm{~h}}$ only. In addition, rather than treating $f_{\text {esc }}$ as a completely free parameter, we determine its value from the profile of ionizing flux, which in turn depends on the neutral gas distribution surrounding galaxies. This effectively renders $f_{\text {esc }}$ and the shape of the one-halo term, which is captured by $u_{\mathrm{n}}(k \mid M)$, dependent on each other.

To derive $u_{\mathrm{n}}(k \mid M)$, we consider the scenario in which ionizing photons are radiated away from the centre of galaxy under the influence of neutral gas distribution in the CGM. While ionizing 
photons escaped into the IGM can also in principle induce largescale fluctuations of the types of nebular emission considered in this work, especially Ly $\alpha$, their strengths are found to be subdominant to the emission close to galaxies (e.g. Cooray et al. 2012b). For the CGM, since a substantial overdensity of neutral hydrogen exists in the circumgalactic environment in the high-redshift universe, the extended Ly $\alpha$ (and other nebular) emission is primarily driven by the luminosity of the ionizing source and the distribution of neutral gas clumps surrounding it. Here, we only provide a brief description of the neutral gas distribution models adopted and refer interested readers to Mas-Ribas \& Dijkstra (2016) and Mas-Ribas et al. (2017) for further details. For the Ly $\alpha$ flux resulting from the fluorescent effect in the CGM, the radial profile at a proper distance $r$ scales as

$\mathrm{d} F_{\mathrm{Ly} \alpha}(r) \propto-r^{-2} f_{\mathrm{c}}(r) f_{\mathrm{esc}}(r) \mathrm{d} r$,

where $r^{-2}$ describes the inverse-square dimming and $f_{\mathrm{esc}}(r)=$ $\exp -\left[\int_{0}^{r} f_{\mathrm{c}}\left(r^{\prime}\right) d r^{\prime}\right]$ represents the fraction of ionizing photons successfully escaped from the ionizing source at distance $r . f_{\mathrm{c}}(r)$ is the differential, radial covering fraction of $\mathrm{H}$ I clumps, whose line-ofsight integral gives the total number of clumps, along a sight line, analogous to the number of mean free path lengths. The product $f_{\mathrm{c}} f_{\mathrm{esc}}$ can be interpreted as the chance that an ionizing photon gets absorbed by a clump of H I cloud and thus gives rise to a Ly $\alpha$ photon. The resulting flux profile can then be expressed as

$F_{\mathrm{Ly} \alpha}(r) \propto \int_{r}^{\infty} r^{\prime-2} f_{\mathrm{c}}\left(r^{\prime}\right) f_{\mathrm{esc}}\left(r^{\prime}\right) \mathrm{d} r^{\prime}$,

given the boundary condition $F_{\mathrm{Ly} \alpha}=0$ as $r \rightarrow \infty$.

Various CGM models have been proposed for high- $z$ galaxies, from which the $\mathrm{H}$ I covering fraction $f_{\mathrm{c}}(r)$ can be obtained. However, due to the paucity of observational constraints especially in the pre-reionization era, it is impractical to robustly determine which one best describes the nebular emission profile of high- $z$ galaxies relevant to our model. As a result, we follow Mas-Ribas et al. (2017) and consider two CGM models that predict distinct HI spatial distributions surrounding galaxies, leading to high and low escape fractions of ionizing photons, respectively. We caution that the two profiles are explored here only to demonstrate the connection between $f_{\text {esc }}(r)$ and small-scale fluctuations. Exact escape fractions they imply are assessed with other observational constraints, such as the CMB optical depth, and therefore some tension may exist for a subset of our Pop III models. We will revisit this point in Section 4.1.

The low-leakage model is based on the fitting formula (see equation 17 of Mas-Ribas \& Dijkstra 2016) for the area covering fraction of Lyman limit systems (LLSs), $\mathcal{F}_{\text {LLS }}$, inferred from the EAGLE simulation (Rahmati et al. 2015). It has been successfully applied to reproduce the observed stacked profile of extended Ly $\alpha$ emission from Lyman-alpha emitters out to $z=6.6$. Specifically, the radial covering fraction $f_{\mathrm{c}}$ is related to the area covering fraction $\mathcal{F}_{\text {LLS }}(b)$, defined for a total area of $2 \pi b \mathrm{~d} b$ at the impact parameter $b$, by an inverse Abel transformation

$f_{\mathrm{c}}(r)=-\frac{1}{\pi} \int_{r}^{\infty} \frac{\mathrm{d} N_{\text {clump }}}{\mathrm{d} y} \frac{\mathrm{d} y}{\sqrt{y^{2}-r^{2}}}$,

where the number of gas clumps encountered is given by $N_{\text {clump }}(b)=$ $-\ln \left[1-\mathcal{F}_{\text {LLS }}(b)\right]$. The high-leakage model is proposed by Steidel et al. (2010) to provide a simple explanation to interstellar absorption lines and Ly $\alpha$ emission in the observed far-UV spectra of Lyman break galaxies at $z \lesssim 3$. It describes a clumpy outflow consisting of cold $\mathrm{H}$ I clumps embedded within a hot medium accelerating radially outward from the galaxy. The radial covering fraction $f_{\mathrm{c}}$ in this case

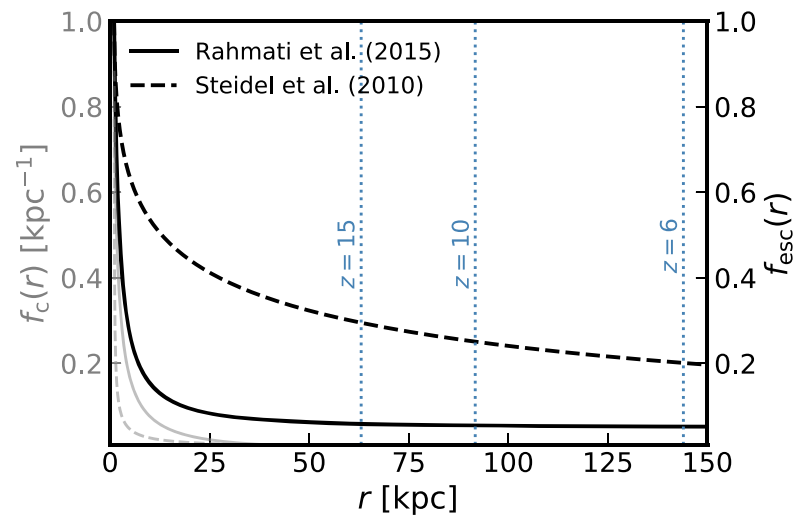

Figure 3. The radial profiles of the $\mathrm{H}$ I covering fraction $f_{\text {esc }}$ (grey, left axis) and the escape fraction of ionizing photons $f_{\text {esc }}$ (black, right axis) as functions of the radial distance $r$ away from the galaxy, derived from two CGM models by Rahmati et al. (2015) and Steidel et al. (2010). The virial radius of a $10^{14} \mathrm{M}_{\odot}$ halo, which defines an upper bound on the scale relevant to ionizing photons escaping into the IGM, is quoted at $z=6,10$, and 15 (dotted vertical lines).

can be written as (Dijkstra \& Kramer 2012)

$f_{\mathrm{c}}(r)=n_{\mathrm{c}}(r) \pi R_{\mathrm{c}}^{2}$,

where $n_{\mathrm{c}}(r)$ is the number density of the HI clumps that is inversely proportional to their radial velocity $v(r)$ determined from the observed spectra, and the clump radius $R_{\mathrm{c}} \propto r^{-2 / 3}$ under pressure equilibrium.

Fig. 3 shows a comparison between radial profiles of $f_{\mathrm{c}}$ and $f_{\text {esc }}$ in the two CGM models considered. The higher H I covering fraction in the Rahmati et al. (2015) model results in an $f_{\text {esc }}$ profile, which declines more rapidly with $r$ than that from the Steidel et al. (2010) model. Given the potentially large uncertainties associated with the exact mapping between the $f_{\text {esc }}$ profile and the average escape fraction $\bar{f}_{\text {esc }}$ that matters for reionization, we refrain from defining $\bar{f}_{\text {esc }}$ at the virial radius of a halo that hosts a typical EoR galaxy, as done by Mas-Ribas et al. (2017). Instead, we quote the value of $f_{\text {esc }}$ as predicted by the two CGM models at a proper distance $r=150 \mathrm{kpc}$, sufficiently large compared to the virial radii of the largest relevant haloes $\left(10^{14} \mathrm{M}_{\odot}\right)$ as shown by the vertical dotted lines in Fig. 3. This allows us to effectively define lower bounds on the average escape fraction $\bar{f}_{\text {esc }}=0.05$ and 0.2 corresponding to the Rahmati et al. (2015) and Steidel et al. (2010) models, respectively, which in turn set upper bounds on the nebular emission signal allowed in the two cases. We note, nevertheless, that both CGM models predict only modest evolution of $f_{\text {esc }}(r)$ beyond a few tens kpc the size range of more typical haloes hosting ionizing sources. The exact choice of $\bar{f}_{\text {esc }}$ value is thus expected to have only a small impact on the NIRB signal predicted, whereas the corresponding reionization history is more sensitive to this choice, as will be discussed in Section 4.1. To simplify the notation, in what follows we will drop the bar and use $f_{\text {esc }}$ to denote the lower bound on $\bar{f}_{\text {esc }}$ inferred from the CGM model chosen. As summarized in Table 1, in our models, we set $f_{\text {esc }}^{\text {III }}=0.05$ or 0.2 for Pop III stars according to the two CGM models, whereas for Pop II stars we adopt an intermediate profile that yields $f_{\text {esc }}^{\mathrm{II}}=0.1$. With reasonable faint-end extrapolations as in our model, an escape fraction of 10 per cent is proven to yield a reionization history consistent with current observations without the presence of unknown source populations like Pop III stars. 


\subsubsection{The angular power spectrum}

Following Fernandez et al. (2010) and Loeb \& Furlanetto (2013), we can derive the angular power spectrum from the 3D power spectrum. With an observed frequency $v$, equation (19) gives the NIRB intensity, which can be expressed as a function of direction on the sky $\hat{\mathbf{n}}$

$I_{\nu}(\hat{\mathbf{n}})=\frac{c}{4 \pi} \int_{z_{\min }}^{z_{\max }} \frac{\epsilon_{v^{\prime}}[z, \hat{\mathbf{n}} r(z)]}{H(z)(1+z)} d z$,

where $v^{\prime}=(1+z) v$ and $r(z)$ is the comoving radial distance out to a redshift $z$. Spherical harmonics decomposing $I_{\nu}(\hat{\mathbf{n}})$ gives

$I_{\nu}(\hat{\mathbf{n}})=\sum_{\ell, m} a_{\ell m} Y_{\ell}^{m}(\hat{\mathbf{n}})$,

with the coefficient

$a_{\ell m}=\frac{c}{4 \pi} \int \frac{\mathrm{d} z \int \mathrm{d} \hat{\mathbf{n}} \int \frac{\mathrm{d}^{3} \mathbf{k}}{(2 \pi)^{3}} \epsilon_{v^{\prime}}(z, \mathbf{k}) e^{-i \mathbf{k} \cdot \hat{\mathbf{n}} r(z)} Y_{\ell m}^{*}(\hat{\mathbf{n}})}{H(z)(1+z)}$.

Using Rayleigh's formula for $e^{-i \mathbf{k} \cdot \hat{\mathbf{n}} r(z)}$, we have

$a_{\ell m}=\int \frac{c(-1)^{\ell} \mathrm{d} z}{H(z)(1+z)} \int \frac{d^{3} \mathbf{k}}{(2 \pi)^{3}} \tilde{\epsilon}_{v^{\prime}}(z, \mathbf{k}) j_{\ell}[k r(z)] Y_{\ell m}^{*}(\hat{\mathbf{k}})$.

The angular power spectrum is consequently defined as the ensemble average $C_{\ell}=\left\langle\left|a_{\ell m}\right|^{2}\right\rangle$. For a pair of observed frequencies $v_{1}$ and $v_{2}$, it can be written as (assuming Limber's approximation, which is valid for the range of $\ell \gg 1$ considered in this work)

$C_{\ell}^{v_{1} v_{2}}=\frac{c}{(4 \pi)^{2}} \int \frac{P_{\mathrm{NIR}}^{v_{1} \nu_{2}}\left[v_{1}(1+z), v_{2}(1+z), \ell / r(z)\right] \mathrm{d} z}{H(z) r^{2}(z)(1+z)^{2}}$,

where $P_{\mathrm{NIR}}^{v_{1} v_{2}}$ is the 3D NIRB power spectrum defined in equation (21).

Alternatively, a band-averaged intensity may be defined, in which case a factor of $(1+z)$ must be introduced to account for the cosmological redshift (Fernandez et al. 2010). Namely, in contrast to equation (29), we have

$$
\begin{aligned}
I(\hat{\mathbf{n}}) & =\frac{1}{\Delta v} \int_{v_{1}}^{v_{2}} \mathrm{~d} \nu I_{v}(\hat{\mathbf{n}}) \\
& =\frac{c}{4 \pi \Delta v} \int \mathrm{d} z \frac{\int_{v_{1}(1+z)}^{\nu_{2}(1+z)} \mathrm{d} \tilde{v} \epsilon_{\tilde{v}}[z, \hat{\mathbf{n}} r(z)]}{H(z)(1+z)^{2}} \\
& =\frac{c}{4 \pi \Delta v} \int \mathrm{d} z \frac{\rho_{L}^{\mathrm{em}}[z, \hat{\mathbf{n}} r(z)]}{H(z)(1+z)^{2}},
\end{aligned}
$$

where $\rho_{L}^{\text {em }}$ represents the luminosity density emitted over some frequency band at the corresponding redshift. The band-averaged angular power spectrum is then

$C_{\ell}=\frac{c}{(4 \pi \Delta v)^{2}} \int \frac{\mathrm{d} z}{H(z) r^{2}(z)(1+z)^{4}} P_{L}^{\mathrm{NIR}}[k=\ell / r(z), z]$.

\section{RESULTS}

In this section, we show the high- $z$ NIRB signals sourced by galaxies at $z>5$, with the emphasis on the potential contribution of Pop III stars. We first present a general picture expected given our reference model which combines a semi-empirical description of the known, Pop II star-forming galaxies and an optimistic model of Pop III star formation, characterized by high Pop III SFR with relatively inefficient chemical feedback (Section 3.1). Then, by exploring a range of plausible Pop III star formation histories, we focus on how spectral signatures of Pop III stars on the NIRB connect to their properties (Section 3.2). Finally, we estimate the sensitivities of two

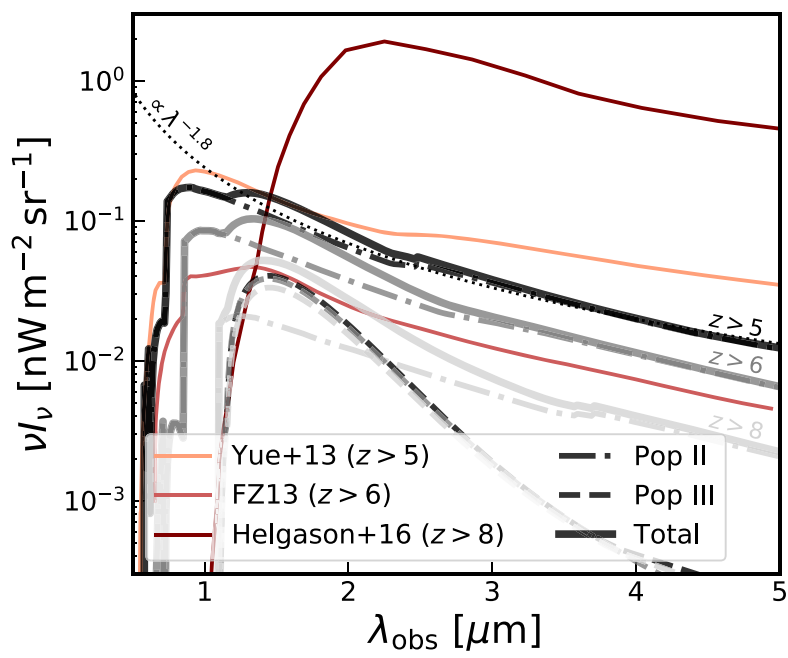

Figure 4. The spectra of NIRB mean intensity $v \bar{I}_{v}$ sourced by Pop II (dashdotted) and Pop III (dashed) star-forming galaxies at different redshifts, predicted by Model IA. The Pop II contribution can be approximated by $\lambda^{-1.8}$. For comparison, we show in colour a few model predictions in the literature that include contributions from both Pop II and Pop III stars (Yue et al. 2013a, FZ13; Helgason et al. 2016). The impact of the Pop III to Pop II transition, which varies significantly among these models, can be seen from the shape and amplitude of NIRB spectrum. A spectral peak redward of 1 micron is characteristic of a significant Ly $\alpha$ contribution to the NIRB intensity due to the efficient formation of massive, Pop III stars.

future instruments, SPHEREx and CDIM, to the high- $z$ NIRB signals (Section 3.3).

\subsection{The NIRB from star-forming galaxies at $z>5$}

To provide a general picture of the NIRB signal associated with first galaxies, we define our reference model to be Model IA, as specified in Table 1 . The SFE of Pop II stars $f_{*}$ follows a double power law in mass fit to the observed galaxy UVLFs over $5<z<10$, and the Pop III SFRD is tuned such that the total cosmic SFRD roughly matches the maximum-likelihood model from Robertson et al. (2015) based on the electron scattering optical depth $\tau_{e}$ of CMB photons from Planck. A set of variations around this baseline case will be considered in the subsections that follow.

In Fig. 4, we show the mean intensity spectra of the NIRB over $0.75-5 \mu \mathrm{m}$, calculated from Model IA with different redshift cutoffs. For comparison, results from the literature that account for both Pop II and Pop III stars with similar cutoffs are also displayed. The sharp spectral break at the Ly $\alpha$ wavelength redshifted from the cutoff is caused by the IGM attenuation as described by Madau (1995), which serves as a characteristic feature that distinguishes the high- $z$ component from low- $z$ ones. From our model, the NIRB spectrum associated with Pop II stars without being blanketed by $\mathrm{H} \mathrm{I}$ blueward of $\mathrm{Ly} \alpha$ is predominantly sourced by direct stellar emission, and it can be well described by a power law that scales as $\lambda^{-1.8}$. This roughly agrees with the Pop-II-dominated prediction from Yue et al. (2013a), who find a slightly shallower slope that might be attributed to different assumptions adopted in the SED modelling and the SFH assumed. Unlike Pop II stars, massive Pop III stars contribute to the NIRB mainly through their nebular emission, especially in Ly $\alpha$. The resulting NIRB spectrum therefore has a much stronger wavelength dependence that traces the shape of the Pop III SFRD. Similar to FZ13, our reference model suggests that strong Ly $\alpha$ emission from 


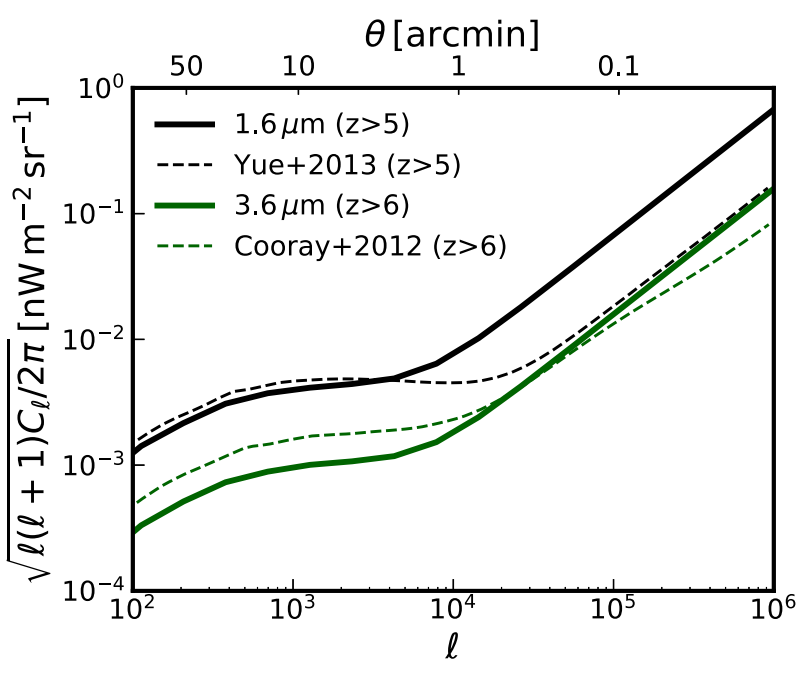

Figure 5. Comparison of the NIRB angular power spectra associated with Pop II and Pop III stars over different bands and redshift ranges. As opposed to Cooray et al. (2012b) and Yue et al. (2013a), our model predicts a higher shot-noise power due to the inefficient star formation in low-mass haloes as described by the mass-dependent $f_{*}$.

Pop III stars may lead to a spectral 'bump' in the total NIRB spectrum, which causes an abrupt change of spectral index over 1-1.5 $\mu \mathrm{m}$. We will discuss the implications of such a Pop III signature in detail in Section 3.2. We also compare our Pop III prediction based on physical arguments of different feedback mechanisms, to an extreme scenario from Helgason et al. (2016) attempting to explain the entire observed, source-subtracted NIRB fluctuations with the Pop III contribution. The fact that our reference model, which already makes optimistic assumptions about the efficiency of Pop III star formation, predicts more than an order of magnitude lower NIRB signal corroborates the finding of Helgason et al. (2016). Pop III stars alone are unlikely to fully account for the observed NIRB excess without violating other observational constraints such as the reionization history - unless some stringent requirements on the physics of Pop III stars are met, including their ionizing and metal production efficiencies.

Fig. 5 shows predicted angular intensity fluctuations $\delta F=$ $\sqrt{\ell(\ell+1) C_{\ell} / 2 \pi}$ of the NIRB by our reference model at two wavelengths, 1.6 and $3.6 \mu \mathrm{m}$. Compared with predictions at the same wavelengths from Cooray et al. (2012b) and Yue et al. (2013a), our model produces similar (within a factor of 2) largescale clustering amplitudes. On small scales, our model predicts significantly higher shot-noise amplitudes. Such a difference in the shape of angular power spectrum, $C_{\ell}$, underlines the importance of properly accounting for the contribution from the population of faint/low-mass galaxies loosely constrained by observations. While all these models assume that haloes above a mass $M_{\min } \sim 10^{8} \mathrm{M}_{\odot}$ can sustain the formation of Pop II stars (which dominates the total NIRB fluctuations) through efficient atomic cooling of gas, our model allows $f_{*}$ to evolve strongly with halo mass. As demonstrated in a number of previous works (Moster et al. 2010; Furlanetto et al. 2017; Mirocha et al. 2017), the observed UVLFs of galaxies at $z$ $>5$ can be well reproduced by $f_{*}$ as a double power law in halo mass, consistent with simple stellar and AGN feedback arguments that suppress star formation in low-mass and high-mass haloes, respectively. Consequently, low-mass haloes in our model, though still forming stars at low levels, contribute only marginally to the observed NIRB fluctuations, especially on small scales where the
Poissonian distribution of bright sources dominates the fluctuations. The resulting angular power spectrum has a shape different from those predicted by Cooray et al. (2012b) and Yue et al. (2013a), with fractionally higher shot-noise amplitude. Measuring the full shape of $C_{\ell}$ from sub-arcminute scales (where the sensitivity to $f_{*}$ maximizes) to sub-degree scales (where the high- $z$ contribution maximizes) with future NIRB surveys can therefore place interesting integral constraints on the effect of feedback regulation on high- $z$, star-forming galaxies, complementary to measuring the faint-end slope of the galaxy UVLF.

\subsection{Spectral signatures of first stars on the NIRB}

As shown in Fig. 4, a characteristic spectral signature may be left on the NIRB spectrum in the case of efficient formation of massive Pop III stars. Details of such a feature, however, depend on a variety of factors involving the formation and physical properties of both Pop II and Pop III stars. Of particular importance is when and for how long the transition from Pop III stars to Pop II stars occurred, which can be characterized by the ratio of their SFRDs, even though stellar physics such as age and the initial mass function (IMF) also matter and therefore serve as potential sources of degeneracy. FZ13 studies the NIRB imprints in this context using a simple phenomenological model for the Pop III to Pop II transition, without considering detailed physical processes that drive the transition. In this subsection, we investigate the effects of varying the Pop II and Pop III SFHs separately on the NIRB signal from high- $z$ galaxies, exploring a set of physically motivated model variations specified in Table 1.

\subsubsection{Effects of variations in the Pop II SFH}

To explore a range of plausible Pop II SFHs, we consider two alternative ways of extrapolating the low-mass end of $f_{*}$ - beyond the mass range probed by the observed UVLFs but still within the constraints of current data - which are labelled as steep and floor, respectively, in table 1 following Mirocha et al. (2017).

In Fig. 6, we show how the level of NIRB intensity fluctuations $\delta F$ and the Pop III signature $\mathcal{R}_{\delta F}=\delta F_{\text {Pop III }} / \delta F_{\text {Pop II }}$ evolve with wavelength, as predicted by the three different combinations of our Pop II SFE models and the reference Pop III model, namely Model IA, Model IIA, and Model IIIA. Values of $\delta F_{\text {Pop II }}$ and $\delta F_{\text {Pop III }}$ are quoted at the centres of the nine SPHEREx broadbands for multipoles $500<\ell<2000$ to facilitate a comparison with the $1 \sigma$ surface brightness uncertainty of SPHEREx in each band, as illustrated by the staircase curve in tan (see Section 3.3 for a detailed discussion of SPHEREx sensitivity forecasts). Overall, the imprint of Pop III stars on the NIRB is connected to (and thus traces) their SFRD evolution through the strong $\operatorname{Ly} \alpha$ emission they produced, with a peak/turnover at the wavelength of Ly $\alpha$ redshifted from the era when Pop III star formation culminated/ended. Near the peak in the $1.5 \mu \mathrm{m}$ band, the NIRB fluctuations contributed by Pop III stars can be up to half as strong as the Pop II contribution. Note that, in practice, the contribution of high- $z$ star-forming galaxies will be blended with other NIRB components from lower redshifts. Separation techniques relying on the distinction in the spectral shape of each component have been demonstrated in e.g. Feng et al. (2019). For reference, we show in Fig. 6 the remaining fluctuation signal associated with low- $z$. $(z \lesssim 3)$ galaxies after masking bright, resolved ones, as predicted by the luminosity function model from Feng et al. (2019). Other sources of emission, such as the IHL may also contribute a significant fraction of the total observed fluctuations - though with a lower certainty, making the component separation even more challenging. 


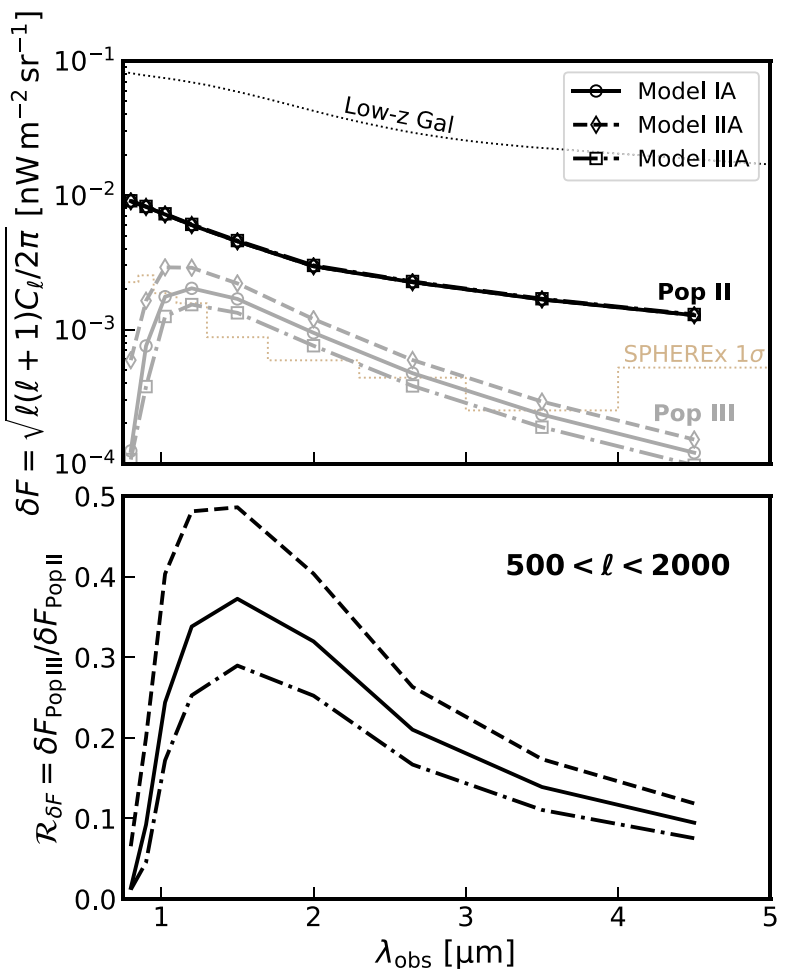

Figure 6. Top: spectra of NIRB intensity fluctuations sourced by $z>5$ starforming galaxies in the angular bin $500<\ell<2000$ predicted by the three variations of the Pop II SFE $f_{*}$ defined in Table 1, compared with the broadband uncertainties of the forthcoming survey in the $200 \mathrm{deg}^{2}$ SPHEREx deep field. Also shown is the expected NIRB fluctuations contributed by low- $z$ galaxies after masking bright resolved sources, taken from Feng et al. (2019). Bottom: the ratio of NIRB intensity fluctuations sourced by Pop III and Pop II stars. The strong evolution with wavelength is driven by the efficient production of Ly $\alpha$ emission by massive Pop III stars.

The effect of varying $f_{*}$ is pronounced for the Pop III contribution, whereas the fluctuations sourced by Pop II stars themselves are barely affected. As discussed in Section 2.1.2 (see also discussion in Mebane et al. 2018), once formed in sufficient number, Pop II stars can play an important role in shaping the Pop III SFH by lifting the minimum mass of Pop III haloes through their LW radiation. The contrast between the steep and floor models suggests that, for a fixed Pop III model, changing $f_{*}$ within the range of uncertainty in UVLF measurements can vary the Pop III signature on the NIRB by up to a factor of two. Unlike the Pop III SFRD, whose dependence on $f_{*}$ grows over time as the LW background accumulates, the dependence of $\mathcal{R}_{\delta F}$ on $f_{*}$ shows only modest evolution with wavelength since Pop III stars formed close to the peak redshift dominate the fluctuation signal at all wavelengths. On the contrary, the Pop II contribution remain almost unaffected by variations of $f_{*}$ because the majority of the fluctuation signal is contributed by Pop II stars at $z \sim 5-6$, which formed mostly in more massive haloes not sensitive to the low-mass end of $f_{*}$ (see Fig. 1).

\subsubsection{Effects of variations in the Pop III SFH}

Apart from the influence of the LW background from Pop II stars, the Pop III SFH is also, and more importantly, determined by the physics of Pop III star formation in minihaloes under the regulation of all sources of feedback. As specified in Table 1, we consider an additional set of three variations of the Pop III star formation
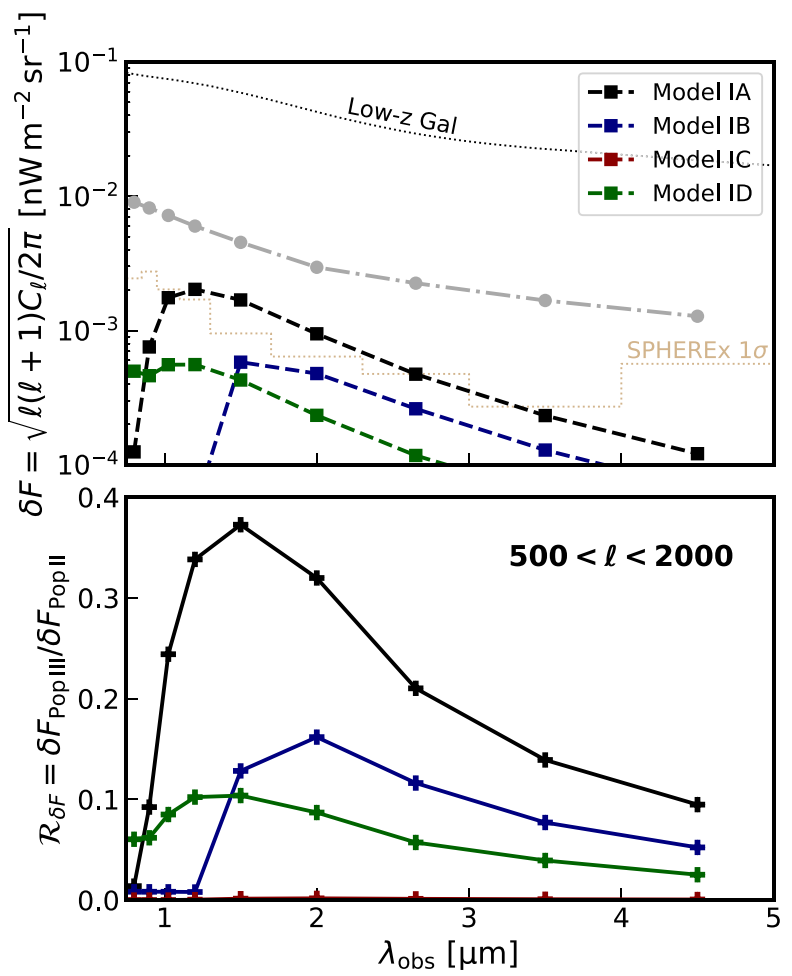

Figure 7. Same as Fig. 6 but for the four variations of the Pop III SFHs defined in Table 1. For comparison, the grey dash-dotted curve shows the Pop II contribution to the fluctuations.

prescription and quantify how the imprint on the NIRB may be modulated.

Similar to Figs 6 and 7 shows the NIRB intensity fluctuations for the four different Pop III models considered, each of which yields a possible Pop III SFH fully regulated by the LW feedback and physical arguments about metal enrichment, as described in Section 2.1.2. Compared with the reference model (Model IA), which implies an extremely high Pop III SFE of order $0.1-1$ by comparing rates of star formation and mass accretion, approximations to the semi-analytic models from Mebane et al. (2018) imply less efficient Pop III star formation and thus predict Pop III SFRDs that are at least one order of magnitude smaller, as illustrated in Fig. 1. Nevertheless, the fluctuation signals in Model IB and ID are only a factor of 2-3 smaller than what Model IA predicts, due to the high mass of Pop III stars assumed in these models which yields a high photoionization rate of $Q(\mathrm{H})=10^{51} \mathrm{~s}^{-1}$. Involving neither a high SFE $\left(\dot{M}_{*}^{\mathrm{III}}=3 \times 10^{-6} \mathrm{M}_{\odot} \mathrm{yr}^{-1}\right)$ nor a very top-heavy IMF $\left(Q(\mathrm{H})=10^{50} \mathrm{~s}^{-1}\right)$, Model IC represents a much less extreme picture of Pop III star formation favoured by some recent theoretical investigations (e.g. Xu et al. 2016a; Mebane et al. 2018), which is unfortunately out of reach for any foreseeable NIRB measurement.

The correspondence between the Pop III SFHs and their spectral signatures on the NIRB can be easily seen by comparing the shapes of $\dot{\rho}_{*}^{\text {III }}(z)$ in Fig. 1 and $\mathcal{R}_{\delta F}$ in the bottom panel of Fig. 7, which suggests that the latter can be exploited as a useful probe for the efficiency and persistence of Pop III formation across cosmic time. In particular, the detailed amplitude of $\mathcal{R}_{\delta F}$ is subject to astrophysical uncertainties associated with, e.g. the stellar SED and escape fraction, which are highly degenerated with the SFH as pointed out by FZ13. However, the contrast between spectra showing turnovers at different redshifts (Model IA versus Model IB), or with or without a spectral 
Table 2. Survey and instrument parameters for SPHEREx deep field and CDIM medium field. Note that the surface brightness sensitivities are quoted at $1.5 \mu \mathrm{m}$ for the $500<\ell<2000$ bin in the last row. The numbers inside the parentheses are the raw surface brightness sensitivities per $\ell$ mode per spectral resolution element, whereas the numbers outside are after spectral and spatial binning.

\begin{tabular}{llcc}
\hline Parameter & Description & SPHEREx & CDIM \\
\hline$A_{\mathrm{S}}\left(\mathrm{deg}^{2}\right)$ & Survey area & 200 & 30 \\
$R$ & Resolving power & 40 & 300 \\
$f_{\text {sky }}$ & Sky coverage & 0.005 & 0.0007 \\
$\Omega_{\text {pix }}(\mathrm{sr})$ & Pixel size & $9.0 \times 10^{-10}$ & $2.4 \times 10^{-11}$ \\
$\sigma_{\text {pix }}\left(\mathrm{nW} / \mathrm{m}^{2} / \mathrm{sr}\right)$ & Sensitivity (SB) & $0.09(1.94)$ & $0.14(24.06)$ \\
\hline
\end{tabular}

break (Model IB versus Model ID), is robust, provided that the aforementioned astrophysical factors do not evolve abruptly with redshift. Any evidence for the existence of such a spectral signature from future facilities like SPHEREx would therefore be useful for mapping the landscape of Pop III star formation. We further elaborate on the prospects for detecting the NIRB signal of Pop III stars in the next subsection.

\subsection{Detecting Pop III stars in the NIRB with SPHEREx and CDIM}

To this point, we have elucidated how massive Pop III stars might leave a discernible imprint on the observed NIRB when formed at a sufficiently high rate $\dot{M}_{*}^{\mathrm{III}} \gtrsim 10^{-3} \mathrm{M}_{\odot} \mathrm{yr}^{-1}$ per minihalo whose minimum mass $M_{h, \text { III }}^{\text {III }}$ is set by the LW feedback, as well as how effects of varying Pop II and Pop III star formation physics can affect such a spectral signature. It is interesting to understand how well the NIRB signal contributed by high- $z$, star-forming galaxies may be measured in the foreseeable future, and more excitingly, what scenarios of Pop III star formation may be probed. For this purpose, we consider two satellites that will be able to study the NIRB in detail, namely SPHEREx (Doré et al. 2014), a NASA MediumClass Explorer (MIDEX) mission scheduled to be launched in 2024, and CDIM (Cooray et al. 2019), another NASA Probe-class mission concept. It is useful to point out that other experiments/platforms also promise to probe the NIRB signal from galaxies duration and before the EoR, including the ongoing sounding rocket experiment CIBER2 (Lanz et al. 2014) and dedicated surveys proposed for other infrared telescopes, such as JWST (Kashlinsky et al. 2015a) and Euclid (Kashlinsky et al. 2015b). In what follows, we focus on the forecasts for SPHEREx and CDIM given their more optimal configurations for NIRB observations, and refer interested readers to the papers listed for details of alternative methods. We note, though, that the high spectral resolution of CDIM (see Table 2) makes 3D line-intensity mapping a likely more favourable strategy for probing first stars and galaxies than measuring $C_{\ell}$, when issues of foreground cleaning and component separation are considered. While in this work we only focus on the comparison of $C_{\ell}$ sensitivities, tomographic Ly $\alpha$ and $\mathrm{H} \alpha$ observations with CDIM and their synergy with 21-cm surveys have been studied (Heneka, Cooray \& Feng 2017; Heneka \& Cooray 2021).

Using the Knox formula (Knox 1995), we can write the uncertainty in the observed angular power spectrum $C_{\ell}$ measured for any two given bands as

$\Delta C_{\ell}=\frac{1}{\sqrt{f_{\text {sky }}(\ell+1 / 2)}}\left(C_{\ell}+C_{\ell}^{\text {noise }}\right)$.
The first term $C_{\ell}$ describes cosmic variance and the second term $C_{\ell}^{\text {noise }}=4 \pi f_{\text {sky }} \sigma_{\text {pix }}^{2} N_{\text {pix }}^{-1} e^{\Omega_{\text {pix }} \ell^{2}}$ is the instrument noise (Cooray et al. 2004), where $N_{\text {pix }}$ is the number of pixels in the survey. At sufficiently large scales where $\ell \ll \Omega_{\text {pix }}^{-1 / 2}$, we have $C_{\ell}^{\text {noise }} \approx \sigma_{\text {pix }}^{2} \Omega_{\text {pix }}$. The prefactor $\left[f_{\text {sky }}(\ell+1 / 2)\right]^{-1 / 2}$ accounts for the number of $\ell$ modes available, given a sky covering fraction of $f_{\text {sky. }}$. To estimate the instrument noise, we take the surface brightness sensitivity estimates made for a total survey area of $200 \mathrm{deg}^{2}$ for SPHEREx and $30 \mathrm{deg}^{2}$ for CDIM, corresponding to the deep- and medium-field surveys planned for SPHEREx and CDIM, respectively. The pixel size $\Omega_{\text {pix }}$ is taken as $9.0 \times 10^{-10} \mathrm{sr}\left(6.2 \times 6.2 \operatorname{arcsec}^{2}\right.$ pixels $)$ and $2.4 \times 10^{-11} \operatorname{sr}(1 \times$ $1 \operatorname{arcsec}^{2}$ pixels) for SPHEREx and CDIM, respectively.

Using the same spectral binning scheme as in Feng et al. (2019), we bin native spectral channels of both SPHEREx and CDIM into the following nine broadbands over an observed wavelength range of $0.75<\lambda_{\text {obs }}<5 \mu \mathrm{m}$ : $(0.75,0.85),(0.85,0.95),(0.95,1.1),(1.1,1.3)$, $(1.3,1.7),(1.7,2.3),(2.3,3.0),(3.0,4.0)$, and $(4.0,5.0)$, regardless of their difference in the raw resolving power $R$ per channel. For the spatial binning of $\ell$ modes, we consider six angular bins over $10^{2}<$ $\ell<10^{6}$ as follows: $\left(10^{2}, 5 \times 10^{2}\right),\left(5 \times 10^{2}, 2 \times 10^{3}\right),\left(2 \times 10^{3}\right.$, $\left.8 \times 10^{3}\right),\left(8 \times 10^{3}, 3 \times 10^{5}\right),\left(3 \times 10^{4}, 1.5 \times 10^{5}\right)$, and $\left(1.5 \times 10^{5}\right.$, $\left.1 \times 10^{6}\right)$, which also apply to both SPHEREx and CDIM, although essentially no information is available on scales smaller than the pixel scale of the instrument. The $N=9$ broadbands specified then allow us to define an angular power spectrum vector $\boldsymbol{C}_{\ell}^{\bar{\lambda}_{\ell} \bar{\lambda}_{2}}$ (for each $\ell$ bin) that consists of $N(N+1) / 2=45$ noise-included, auto- and crosspower spectra measurable from the broadband images. As shown in Table 2, even though the surface brightness (SB) sensitivity per pixel of the CDIM medium field (T.-C. Chang, private communication) is comparable to that of the SPHEREx deep field ${ }^{7}$ after binning, its band noise power $C_{\ell}^{\text {noise }}$ is in fact an order of magnitude lower thanks to CDIM's much smaller pixel size. For simplicity, we assume that the noise contribution from maps of different bands is uncorrelated, such that entries of the noise-included vector $\tilde{\boldsymbol{C}}_{\ell}^{\bar{\lambda}_{1} \bar{\lambda}_{2}}$ can be expressed as $C_{\ell}^{\bar{\lambda}_{1} \bar{\lambda}_{2}}+\delta_{\bar{\lambda}_{1} \bar{\lambda}_{2}} C_{\ell}^{\bar{\lambda}_{1} \bar{\lambda}_{2} \text {,noise }}$, which is distinguished from the signal-only vector $\boldsymbol{C}_{\ell}^{\bar{\lambda}_{1} \bar{\lambda}_{2}}$ by the Kronecker delta $\delta_{\bar{\lambda}_{1} \bar{\lambda}_{2}}$.

The resulting signal-to-noise ratio $(\mathrm{S} / \mathrm{N})$ of the full-covariance measurement (summed over all angular bins of $\ell$ )

$$
\left(\frac{S}{N}\right)^{2}=\sum_{\ell}\left(\boldsymbol{C}_{\ell}^{\bar{\lambda}_{1} \bar{\lambda}_{2}}\right)^{\mathrm{T}}\left(\boldsymbol{C}_{\ell, \mathrm{COV}}^{\bar{\lambda}_{1} \bar{\lambda}_{2}, \bar{\lambda}_{1}^{\prime} \bar{\lambda}_{2}^{\prime}}\right)^{-1}\left(\boldsymbol{C}_{\ell}^{\bar{\lambda}_{1}^{\prime} \bar{\lambda}_{2}^{\prime}}\right)
$$

is then used to quantify the detectability of the NIRB signals by the two surveys considered. Here, the covariance matrix between two band power spectra $\boldsymbol{C}_{\ell}^{\bar{\lambda}_{1} \bar{\lambda}_{2}}$ and $\boldsymbol{C}_{\ell}^{\bar{\lambda}_{1}^{\prime} \bar{\lambda}_{2}^{\prime}}$ can be expressed using Wick's theorem as (Feng et al. 2019)

$\boldsymbol{C}_{\ell, \operatorname{COV}}^{\bar{\lambda}_{1} \bar{\lambda}_{2}, \bar{\lambda}_{1}^{\prime} \bar{\lambda}_{2}^{\prime}}=\frac{1}{f_{\text {sky }}(2 \ell+1)}\left[\tilde{C}_{\ell}^{\bar{\lambda}_{1} \bar{\lambda}_{1}^{\prime}} \tilde{C}_{\ell}^{\bar{\lambda}_{2} \bar{\lambda}_{2}^{\prime}}+\tilde{C}_{\ell}^{\bar{\lambda}_{1} \bar{\lambda}_{2}^{\prime}} \tilde{C}_{\ell}^{\bar{\lambda}_{1}^{\prime} \bar{\lambda}_{2}}\right]$,

which reduces to equation (36) when $\bar{\lambda}_{1}=\bar{\lambda}_{1}^{\prime}=\bar{\lambda}_{2}=\bar{\lambda}_{2}^{\prime}$.

In Table 3, we summarize the raw sensitivities to $C_{\ell}$ in terms of the total S/N that SPHEREx and CDIM are expected to achieve in the four different Pop III star models considered in this work. Since the contribution from Pop II stars dominates over that from Pop III stars at all wavelengths except where the Pop III signature appears $(\sim 1.5 \mu \mathrm{m})$, a significantly higher raw $\mathrm{S} / \mathrm{N}$ is expected for the

\footnotetext{
${ }^{7}$ See the public product for projected surface brightness sensitivity levels of SPHEREx at https://github.com/SPHEREx/Public-products/blob/master/Sur face_Brightness_v28_base_cbe.txt.
} 
Table 3. The estimated raw S/N of NIRB signals sourced by Pop II and Pop III star-forming galaxies at $z>5$, using only auto-power spectra measured in the nine broadbands or all 45 available auto- and cross-power spectra combined. For each entry, the first and second numbers represent the S/N estimated for SPHEREx deep survey and CDIM medium survey, respectively.

\begin{tabular}{lcccc}
\hline Model & $(\mathrm{S} / \mathrm{N})_{\text {Pop II }}^{\text {auto }}$ & $(\mathrm{S} / \mathrm{N})_{\text {Pop III }}^{\text {auto }}$ & $(\mathrm{S} / \mathrm{N})_{\text {Pop II }}^{\text {all }}$ & $(\mathrm{S} / \mathrm{N})_{\text {Pop III }}^{\text {all }}$ \\
\hline IA & $68 / 1100$ & $8.8 / 86$ & $120 / 2300$ & $13 / 110$ \\
IB & $68 / 1100$ & $1.9 / 38$ & $120 / 2300$ & $2.8 / 45$ \\
IC & $68 / 1100$ & $0.0 / 1 \times 10^{-3}$ & $120 / 2300$ & $0.0 / 2 \times 10^{-3}$ \\
ID & $68 / 1100$ & $0.8 / 6.0$ & $120 / 2300$ & $1.4 / 10$ \\
\hline
\end{tabular}

former, reaching above 100 when combining all the auto- and crosscorrelations available and summing up all angular bins for SPHEREx, similar to what was previously found by Feng et al. (2019). For Pop III stars, our optimistic Model IA predicts a raw S/N greater than 10 for SPHEREx, which is dominated by the first three angular bins with $\ell \lesssim 10^{4}$, whereas more conservative models assuming lower Pop III SFR per halo predict much smaller raw $\mathrm{S} / \mathrm{N}$ of only a few. Compared with SPHEREx, CDIM is expected to provide approximately a factor of 20 (10) improvement on the total (Pop III) raw S/N achievable, thanks to the competitive SB sensitivity at its small pixel size. This allows CDIM to measure the Pop III contribution at the same significance (S/N 100) as the Pop II contribution for SPHEREx in Model IA when the full covariance is leveraged. We note, though, that in practice the contribution from high- $z$, star-forming galaxies must be appropriately separated from all other components of the source-subtracted NIRB, such as unsolved low- $z$ galaxies, the IHL and the diffuse Galactic light, which lead to a significant reduction of the constraining power on the high- $z$ component (Feng et al.
2019). This component separation issue will be discussed further in Section 5.2.

We show in the left-hand panel of Fig. 8 a comparison of the auto-correlation angular power spectra $C_{\ell}$ of the NIRB predicted by our models in the $1.5 \mu \mathrm{m}$ band. For clarity, we only show the Pop II signal in Model IA (dash-dotted curve) since it hardly varies with the model variations considered. For Pop III stars, a subset of models yielding NIRB signals potentially detectable for SPHEREx and/or CDIM are shown by the dashed curves with varying thickness and colour. The pessimistic Model IC is rescaled and then plotted for completeness. The right-hand panel of Fig. 8 illustrates the effect of changing Pop II and Pop III models on the shape of $C_{\ell}$ by showing the ratio of intensity fluctuations $\mathcal{R}_{\delta F}$, which is used to characterize the Pop III signature in Section 3.2, as a function of $\ell$. In all models, $\mathcal{R}_{\delta F}$ peaks at around $\ell \sim 10^{3}$ or an angular scale of $\sim 10$ arcmin, similar to what was found by e.g. Cooray et al. (2004). The fact that in cases like Model IB the fluctuations are preferentially stronger on large angular scales compared to Model IA is because, in the former case, Pop III stars formation completed at much higher redshift and thus was more clustered.

In Fig. 9, we further show the halo-model compositions (i.e. onehalo, two-halo, and shot-noise terms) of $C_{\ell}$ in each Pop III model. Moreover, two possible forms of the one-halo profile motivated by the CGM models, as described in Section 2.4 and Fig. 3, are displayed for the Pop III contribution.

Three notable features show up from this decomposition of $C_{\ell}$. First, the relative strengths of the one-halo component $C_{\ell}^{1 \mathrm{~h}}$ and shot-noise component $C_{\ell}^{\text {shot }}$ are distinct for Pop II and Pop III stars. Because the nebular emission is subdominant to the stellar emission for Pop II stars, on small angular scales their one-halo term is
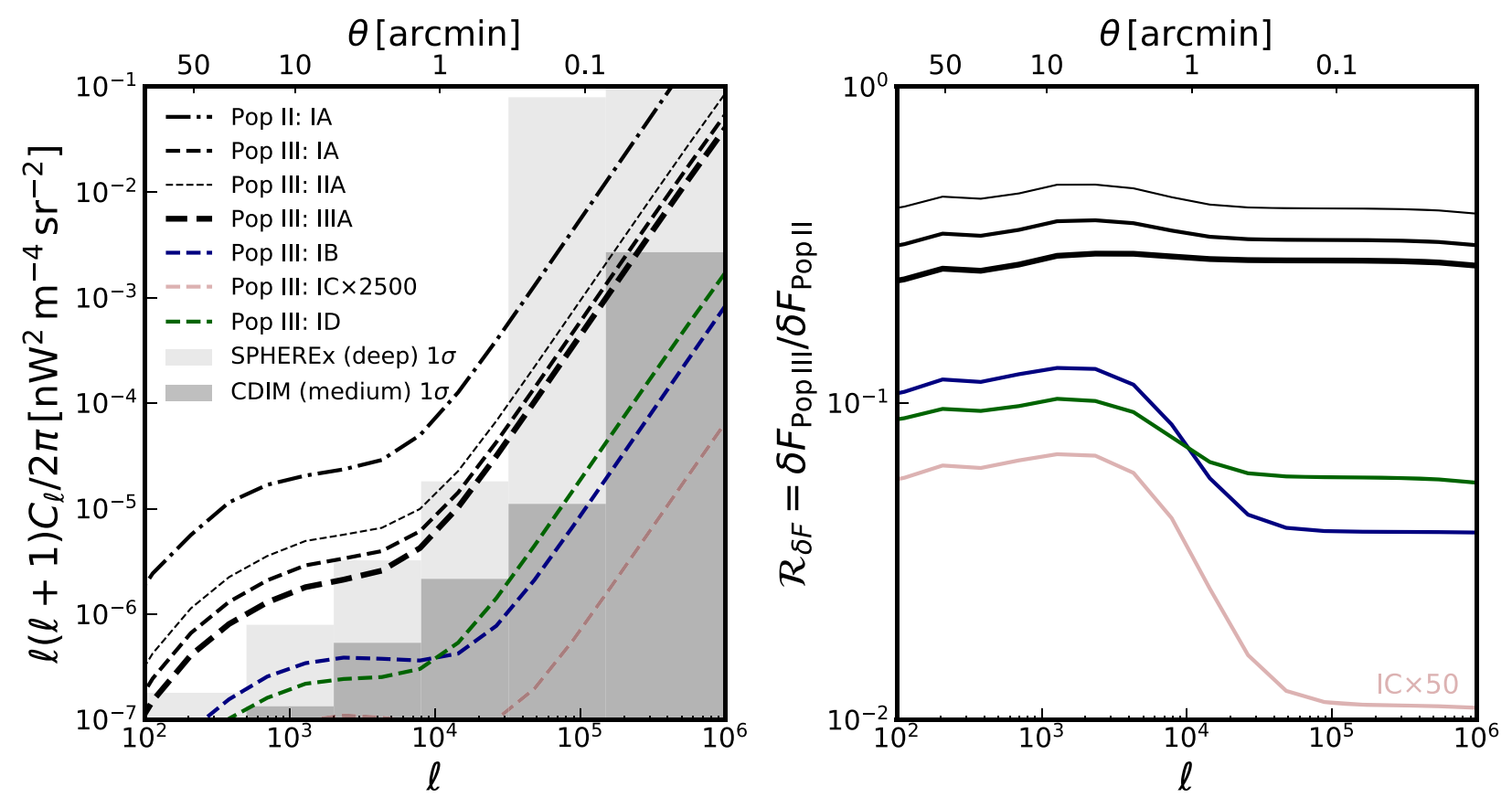

Figure 8. Left: the angular auto-power spectrum $C_{\ell}$ of the NIRB at $1.5 \mu \mathrm{m}$ predicted by different combinations of Pop II and Pop III models. Contributions from Pop II and Pop III stars are shown by dash-dotted and dashed curves, respectively. Variations of Pop II model with steep, dpl, and floor SFE are represented by the thin, intermediate, and thick curves, respectively, whereas different colors represent different Pop III variations. The prediction of Model IC is raised by a factor of 2500 (50) to fit in the left-hand (right) panel. The light and dark shaded regions indicate the expected band uncertainties of SPHEREx deep and CDIM medium surveys, respectively, after binning spectral channels and multipoles according to the imaging broadbands and angular bins defined (see text). Note that the band uncertainty of SPHEREx in the largest $\ell$ bin goes to infinity since such small scales are inaccessible, given the pixel size of SPHEREx. Right: the ratio of NIRB intensity fluctuation amplitudes of Pop III and Pop II stars as a function of multipole moment $\ell$. 

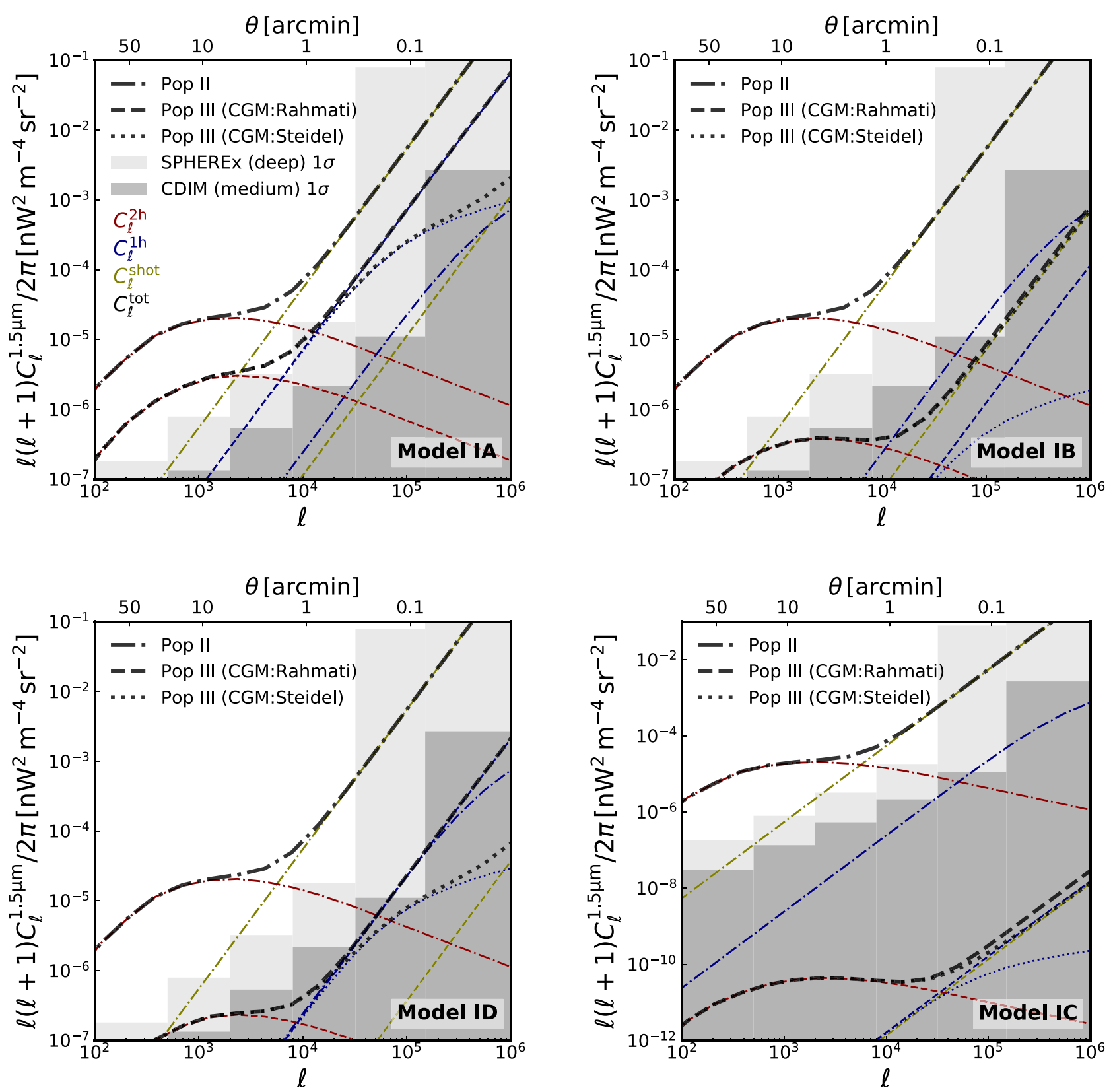

Figure 9. Contributions from $z>5$ Pop II and Pop III star-forming galaxies to the two-halo, one-halo, and shot-noise components of $C_{\ell}$ measured at $1.5 \mu$ m. Clockwise from the top left-hand panel: the figures show $C_{\ell}$ predicted by Model IA, Model IB, Model IC, and Model ID, defined in Table 1. In each panel, the one-halo term is shown for two instances of CGM profile to illustrate the connection between the escape of ionizing photons and the shape of the one-halo term. The light and dark shaded regions indicate the expected band uncertainties of SPHEREx deep and CDIM medium surveys, respectively, after binning spectral channels and multipoles according to the imaging broadbands and angular bins defined (see text). Note the different $y$-axis scale used in the bottom right panel to show the Pop III signal.

negligible compared to the shot-noise term, making $C_{\ell}$ of Pop II stars almost scale-invariant at $\ell>10^{4}$. On the contrary, Pop III stars can produce very strong nebular emission, especially Ly $\alpha$, which makes it possible for their one-halo term to dominate on small angular scales. Such an effect can be seen in the left two panels of Fig. 9, where the one-halo term is approximately 1.5 dex higher than the shot-noise term.

Secondly, amplitudes of the one-halo and shot-noise components also depend on the exact SFH, or more specifically, the persistence of Pop III star formation. As shown by the contrast between the left- hand and right-hand two panels of Fig. 9, models with an extended Pop III SFH (but not necessarily a later Pop III to Pop II transition, see Fig. 1) that persists till $z<10$ provide the nebular emission with sufficient time to overtake the stellar emission in the contribution to the NIRB, thereby resulting in a stronger one-halo term.

Last but not least, we leverage the physical picture illustrated in Fig. 3 to enable additional flexibility in the modelling of the one-halo term by physically connecting its profile with the escape fraction of ionizing photons $f_{\text {esc }}^{\mathrm{III}}$. Taking the two CGM models considered and described in Section 2.4, we get two distinct profiles corresponding 
to (lower limits on) escape fractions of 5 per cent and 20 per cent, respectively. When the one-halo term is strong enough on scales of $\ell>10^{4}$, e.g. in Model IA or ID, such a difference in the radiation profile leads to a clear distinction in the shape of the total power spectrum on these scales. This can be seen by comparing the dashed and dotted curves in black in Fig. 9, with a more scale-dependent one-halo term corresponding to a more extended profile of ionizing flux and thus higher escape fraction. It is useful to note that, in most cases considered in this work, an escape fraction of 20 per cent for Pop III stars ends up with a reionization history too early to be consistent with the CMB optical depth constraint from the Planck polarization data, as we will discuss in the next section. Nevertheless, we consider that the two values of $f_{\mathrm{esc}}^{\mathrm{III}}$ chosen are plausible, allowing us to demonstrate how constraints on small-scale fluctuations, in particular the detailed shape of $C_{\ell}^{1 \mathrm{~h}}$, that SPHEREx and CDIM are likely to place may shed light on the escape of ionizing photons from the first ionizing sources at $z \gtrsim 10$.

To this point, we have shown how detectable the high- $z$ contribution from Pop II and Pop III stars to the NIRB would be when compared with sensitivity levels achievable by upcoming/proposed instruments. An important question that follows is how to separate this high- $z$ component from others and, preferably, disentangle the Pop II and Pop III signals. Without the input of external data sets, such as another tracer of star-forming galaxies to be cross-correlated with the key idea of the solution lies in the utilization of the distinctive spatial and spectral structures of different components. As shown in Fig. 4, the high- $z$ component dominated by Pop II stars is characterized by a Lyman break due to the blanketing effect of intergalactic HI. Such a spectral feature has been demonstrated to be useful for isolating the high- $z$ component from sources from lower redshifts (e.g. Feng et al. 2019). Similar ideas apply to the separation of the much weaker Pop III signal from the Pop II signal, thanks to distinctions in their wavelength dependence (due to different types of emission dominating Pop II and Pop III signals, see Fig. 7) and angular clustering (due to different halo mass and redshift distributions of Pop II and Pop III signals, see Fig. 8). Despite an extremely challenging measurement, these contrasts in spatial and spectral structures make it possible, at least in principle, to distinguish templates of the high- $z$ component as a whole or Pop II and Pop III signals separately. We will elaborate on this component separation issue further in Section 5.2, although a detailed study of it is beyond the scope of this paper and thus reserved for future work.

\section{IMPLICATIONS FOR OTHER OBSERVABLES}

Probing ionizing sources driving the EoR with an integral and statistical constraint like the NIRB has a number of advantages compared to the observation of individual sources, including lower cost of observing time, better coverage of the source population, and importantly, synergy with other observables of the EoR. Taking our models of high- $z$ source populations for the NIRB, we discuss in this section possible implications for other observables, such as the reionization history and $21-\mathrm{cm}$ signal, that can be made from forthcoming NIRB measurements.

\subsection{Reionization history}

In the left-hand panel of Fig. 10, we show reionization histories, characterized by the volume-averaged ionized fraction of the IGM, that our models of Pop II/III star formation predict under two different assumptions of the escape fraction $f_{\mathrm{esc}}^{\mathrm{III}}$, namely 5 per cent and 20 per cent derived from the CGM models by Rahmati et al. (2015) and Steidel et al. (2010), respectively. We note that to compute the reionization history, we assume a constant escape fraction of $f_{\text {esc }}^{\mathrm{II}}=10$ per cent for Pop II stars, which is known to yield a $\tau_{e}$ in excellent agreement with the best-estimated value based on the latest Planck data (e.g. Pagano et al. 2020) without Pop III contribution. The middle panel of Fig. 10 shows contributions to the total $\tau_{e}$ at different redshifts calculated from the reionization histories predicted. Among the four models shown, Model IA forms Pop III stars too efficiently to reproduce the $\tau_{e}$ constraint from Planck, even with $f_{\text {esc }}^{\text {III }}$ as low as 5 per cent. To reconcile this tension, we include an additional case setting $f_{\text {esc }}^{\text {III }}$ to 1 per cent as shown by the red dotted curve, which yields a $\tau_{e}$ value marginally consistent with the Planck result. We stress that the LyC escape fraction of Pop III galaxies is poorly understood. A 'radiation-bounded' picture of the escape mechanism generally expects an higher escape fraction than Pop II galaxies, due to the extremely disruptive feedback of Pop III stars (Xu et al. 2016b). A 'density-bounded' picture, however, requires the ionized bubble to expand beyond the virial radius, and thus predicts significantly lower LyC escape fraction for relatively massive $\left(M_{h} \gtrsim 10^{6.5} \mathrm{M}_{\odot}\right)$ minihaloes where the majority of Pop III stars formed (e.g. Tanaka \& Hasegawa 2021). Therefore, besides $\tau_{e}$, which is arguably the most trusted observable, NIRB observations provide an extra handle on jointly probing the SFR and escape fraction of minihaloes forming Pop III stars.

In general, earlier reionization is expected for a model that predicts stronger Pop III star signature on the NIRB, and in Model IB, where the Pop III to Pop II transition is early and rapid, unusual double reionization scenarios can even occur. A caveat to keep in mind, though, is that certain forms of feedback, especially photoheating, which are missing from our model can actually alter the chance of double reionization by affecting the mode and amount of star formation in small haloes, making double reionization implausible (Furlanetto \& Loeb 2005). As such, we refrain from reading too much into this double reionization feature, which is likely due to the incompleteness of our modelling framework, and focus on the integral measure $\tau_{e}$ instead. While it is challenging to establish an exact mapping between the NIRB signal and reionization history, detecting a Pop III signal as strong as what Model IB or ID predicts would already provide tantalizing evidence for a nontrivial contribution to the progression of reionization from Pop III stars. Such a high- $z$ tail for reionization may be further studied through more precise and detailed measurements of NIRB imprints left by Pop III stars, or via some alternative and likely complementary means such as the kSZ effect (e.g. Alvarez et al. 2021) and the E-mode polarization of CMB photons (e.g. Qin et al. 2020; Wu et al. 2021). Also worth noting is that, in order not to overproduce $\tau_{e}$, in cases where the Pop III signature is nontrivial the escape fraction must be either restricted to a sufficiently small upper bound, or allowed to evolve with halo mass and/or redshift. Such constraints on the form of $f_{\mathrm{esc}}^{\mathrm{III}}$ would become more stringent for a stronger NIRB signature, as indicated by the curves in different colors and line styles in the middle panel of Fig. 10. Combining measurements of $C_{\ell}^{1 \mathrm{~h}}$ on sub-arcmin scales with observations of the EoR history, we find it possible to constrain the budget of ionizing photons from Pop III stars, especially $f_{\text {esc }}^{\text {III }}$.

\subsection{The 21-cm signal}

We show in the right-hand panel of Fig. 10 the 21-cm global signal, i.e. the sky-averaged differential brightness temperature of the 21$\mathrm{cm}$ line of neutral hydrogen, implied by each of our Pop III star formation models. Similar to what is found by Mirocha et al. (2018), 

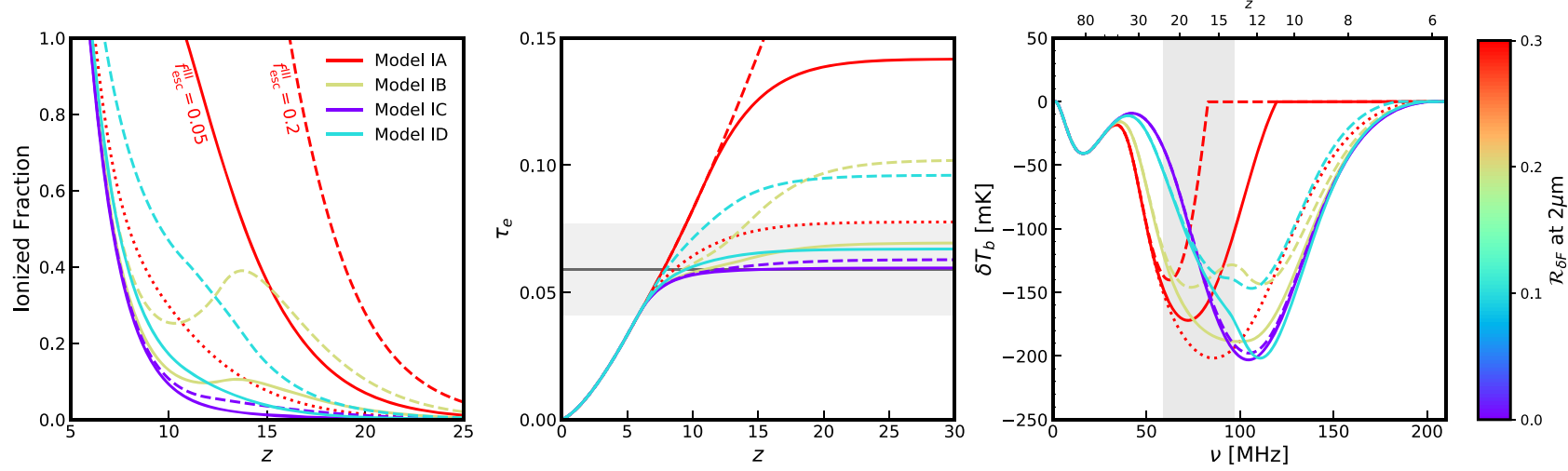

Figure 10. Left: the impact of Pop III stars on the reionization history and NIRB fluctuations. Different line styles represent different assumptions of $f_{\text {esc }}^{\text {III }}$, with an additional dotted curve showing the case of $f_{\text {esc }}^{\mathrm{III}}=0.01$ for Model IA. Curves are colour-coded by the Pop III signature $\mathcal{R}_{\delta F}$ at $2 \mu \mathrm{m}$, where the models can be best distinguished from each other. Middle: the electron scattering optical depth $\tau_{e}$ implied by each model. The horizontal line and grey shaded region indicate the $3 \sigma$ confidence interval on $\tau_{e}$ inferred from CMB polarization data measured by Planck (Pagano et al. 2020). Right: the 21-cm global signal $\delta T_{b}$ implied by each model. The grey shaded region indicates the width of the global signal peaking at $78 \mathrm{MHz}$ as measured by EDGES (Bowman et al. 2018).

models with efficient formation of massive Pop III stars, which leave discernible imprints on the NIRB, predict qualitatively different 21$\mathrm{cm}$ global signals from that predicted by a baseline model without significant Pop III formation (e.g. Model IC). Except for cases with unrealistically early reionization, Pop III stars affect the lowfrequency side of the global signal the most, modifying it into a broadened and asymmetric shape that has a high-frequency tail. The absorption trough gets shallower with increasing Pop III SFR and/or $f_{\text {esc }}^{\mathrm{III}}$, as a result of enhanced heating by the $\mathrm{X}$-rays and a lower neutral fraction.

A tentative detection ${ }^{8}$ of the $21-\mathrm{cm}$ global signal was recently reported by the Experiment to Detect the Global Epoch of Reionization Signature (EDGES; Bowman et al. 2018), which suggests an absorption trough centered at $78.1 \mathrm{MHz}$, with a width of $18.7 \mathrm{MHz}$ and a depth of more than $-500 \mathrm{mK}$. Regardless of the absorption depth, which may only be explained by invoking some new cooling channels of the IGM or some additional radio sources (than the $\mathrm{CMB}$ ) in the early universe, a peak centering at $78.1 \mathrm{MHz}$ is beyond the expectation of simple Pop II-only models based on extrapolations of the observed galaxy UVLFs (Mirocha \& Furlanetto 2019). Additional astrophysical sources, such as Pop III stars may help provide the early Wouthuysen-Field (WF) coupling effect and $\mathrm{X}$-ray heating required to explain the absorption at $78.1 \mathrm{MHz}$, as shown in the right-hand panel of Fig. 10 by the shift of curves towards lower frequencies (see also Mebane et al. 2020). Therefore, insights into the Pop III SFH from NIRB observations would be highly valuable for gauging how much the tension between the EDGES signal and galaxy model predictions might be reconciled by including the contribution of Pop III stars.

Besides the global signal, fluctuations of the $21-\mathrm{cm}$ signal also serves as an important probe of reionization. Various physical properties of Pop III stars are expected to be revealed through their effects on cosmic 21-cm power spectrum, especially the timings of the three peaks corresponding to WF coupling, X-ray heating, and reionization (Mebane et al. in preparation). On the other hand, the cross-correlation between $21 \mathrm{~cm}$ and NIRB observations has been

\footnotetext{
${ }^{8}$ Note, however, that concerns remain about the impact of residual systematics such as foreground contamination on the EDGES results (see e.g. Draine \& Miralda-Escudé 2018; Hills et al. 2018; Bradley et al. 2019; Sims \& Pober 2020).
}

discussed in a few previous works as a way to trace the reionization history (e.g. Fernandez et al. 2014; Mao 2014). We will investigate how to develop a much deeper understanding of Pop III star formation from synergies of $21 \mathrm{~cm}$ and NIRB data in future work.

\section{DISCUSSION}

\subsection{Limitations and the sensitivity to model assumptions}

So far, we have described a semi-empirical model of the high- $z$ NIRB signal, based on physical arguments of Pop II and Pop III star formation calibrated against latest observations of high- $z$ galaxies. Our modelling framework, however, is ultimately still simple in many ways. While more detailed treatments are beyond the scope of this paper and thus left for future work, in what follows, we discuss some major limitations of our model, together with how our findings might be affected by the simplified assumptions.

A key limitation of our model is its relatively simple treatment of the emission spectra of source populations. Despite that (i) the Pop II SED is modelled with the SPS, assuming the simplest possible composite stellar population with a constant SFH, and (ii) the Pop III SED can be reasonably approximated as a blackbody, certain aspects of the complicated problem are unaccounted. These include choices of the IMF, stellar metallicity (for Pop II stars only) and age, etc. and their potential redshift evolution, as well as effects of the stochasticity among galaxies, the extinction by dust, and so forth. We expect our main results about Pop III stars, phrased in terms of a 'perturbation' to the Pop II-only baseline scenario, to be robust against these sources of complexity, even though quantifying their exact effects on the shape and amplitude of high- $z$ NIRB signals would be highly valuable in the near future.

Another important limitation is associated with free parameters that are loosely connected to the physics of source populations, such as the nuisance parameters defining the shape of $f_{*}$, escape fractions of LyC and LW photons, and parameters $\mathcal{T}_{c}$ and $\mathcal{E}_{c}$ used to set the efficiency and persistence of Pop III star formation. While making it easy to explore a wide range of possible scenarios of star formation and reionization, these parameters may not represent an ideal way to parametrize the high- $z$ NIRB signal, meaning that they can be oversimplified or physically related to each other and other implicit model assumptions such as the IMF in practice. Either 
way, unwanted systematics and degeneracy could arise, making data interpretation with the model challenging and less reliable. Looking ahead, we find it useful to develop a more unified (but still flexible) framework for parametrizing the NIRB, identifying and reflecting the connections among physical quantities/processes of interest. This will be particularly useful for parameter inference in the future.

\subsection{Component separation of the observed NIRB}

As already mentioned at the end of Section 3.3, an important challenge in the NIRB data analysis is the separation of its components, which have a broad range of astrophysical origins (Kashlinsky et al. 2018). Failing to perform component separation properly and effectively will make it impossible to constrain a component as weak as the signal from high- $z$ galaxies. Fortunately, as demonstrated in Feng et al. (2019), by measuring the full-covariance angular power spectrum of the observed NIRB, one can reliably separate the major components thanks to their different spatial and spectral structures. In the presence of much stronger low- $z$ components, this approach allows the contribution from EoR galaxies to be recovered and constrained with sufficient significance $(S / N \gtrsim 5)$, without the need for external data sets. To actually reveal the formation histories of the first stars, one must also tell apart the contributions of Pop II and Pop III stars. In addition to the similar full-covariance method discussed in Section 3.3, which makes use of the spectral and spatial differences of Pop II and Pop III signals, it can be also promising to consider a joint analysis with ancillary data. External data sets such as 21-cm maps (e.g. Cox et al., in preparation) and galaxy distributions (e.g. Scott, Upton Sanderbeck \& Bird 2021) can be useful resources for cross-correlation analyses, which are expected to be available from observatories, such as HERA (DeBoer et al. 2017), SKA (Mellema et al. 2013), and the Roman Space Telescope (Spergel et al. 2015) in the coming decade. While tracers like the $21-\mathrm{cm}$ signal and photometric galaxies are also complicated by foregrounds and/or survey-specific systematics, which cause loss of information in inaccessible modes, the extra redshift information from crosscorrelating the NIRB with these 3D tracers makes the problem of separating the high- $z$ component more tractable.

\section{CONCLUSIONS}

In this work, we develop the modelling framework for the NIRB signals sourced by Pop II and Pop III star-forming galaxies at $z$ $>5$. We leverage a semi-empirical approach to build our model on top of physically motivated prescriptions of galaxy evolution and star formation under feedback regulation, and calibrate them to observations of high- $z$ galaxies. Using our model, we analyse how the formation histories of first stars may be revealed by measuring the spatial and spectral properties of the NIRB.

Our main findings can be summarized as follows:

(i) Using a collection of variations in Pop II and Pop III SFHs derived from our model, we reinforce the modelling of the contribution to the NIRB from high- $z$ star-forming galaxies by characterizing the dependence of its shape and amplitude on physics of star formation and galaxy evolution. We find little difference in the predicted contribution of Pop II stars to the NIRB, given the uncertainty in the SFE allowed by constraints on the faint-end slope of galaxy UVLFs. The Pop III SFH, on the contrary, is highly uncertain and sensitive to the LW feedback from both Pop II and Pop III stars themselves, leading to substantial variations in their imprints on the NIRB. (ii) Depending on exact SFHs and detailed properties of Pop III stars such as the IMF, they are expected to leave characteristic spectral signatures on the NIRB at wavelengths redward of $1 \mu \mathrm{m}$ due to their strong Ly $\alpha$ emission. In our optimistic models with efficient formation of massive Pop III stars, such signatures can be as strong as up to a few tens of percent of the fluctuations sourced by Pop II stars, making the NIRB a promising probe of the first stars. Spatial information of the NIRB, such as the shape of the power spectrum, can also shed light on the physics of the first stars, including effects of various feedback processes and the escape of LyC photons.

(iii) Forthcoming space missions like SPHEREx and CDIM can quantify the NIRB fluctuations contributed by high- $z$ galaxies, and thereby placing interesting constraints on the Pop III SFH that is difficult to measure by observing individual galaxies. Even though only optimistic models where massive Pop III stars of $\gtrsim 100 \mathrm{M}_{\odot}$ form at a high efficiency of order $0.1-1$ in minihaloes (resulting in a peak Pop III SFRD as high as $\sim 10^{-3} \mathrm{M}_{\odot} \mathrm{yr}^{-1} \mathrm{Mpc}^{-3}$, or $\dot{M}_{*}^{\text {III }} \sim 10^{-3} \mathrm{M}_{\odot} \mathrm{yr}^{-1}$ in individual minihaloes) may be probed in the SPHEREx deep field, ruling out or disfavouring such extremely scenarios with SPHEREx would still be extremely interesting. With better surface brightness sensitivity, the CDIM medium-field survey has the better chance to inspect a larger subset of plausible Pop III models with less efficient star formation and/or less top-heavy IMFs.

(iv) Any constraints on the first stars from NIRB measurements can have interesting implications for other EoR observables, including the global reionization history, 21-cm signal, and the CMB. In the future, joint analyses of all these probes will provide the best opportunity for overcoming observational systematics such as foreground contamination and studying the first stars from an angle different from, and complementary to, the traditional approach of observing individual galaxies.

\section{ACKNOWLEDGEMENTS}

The authors would like to thank Lluis Mas-Ribas for providing updated models of extended Ly $\alpha$ emission and comments on the early draft, as well as Jamie Bock, Tzu-Ching Chang, Asantha Cooray, Olivier Doré, Chang Feng, Caroline Heneka, and Adam Lidz for helpful discussion about SPHEREx and CDIM instruments and scientific implications. GS is indebted to David and Barbara Groce for the provision of travel funds. JM acknowledges support from a CITA National fellowship. SRF was supported by the National Science Foundation through award AST-1812458. In addition, SRF was directly supported by the NASA Solar System Exploration Research Virtual Institute cooperative agreement number 80ARC017M0006. SRF also acknowledges a NASA contract supporting the 'WFIRST Extragalactic Potential Observations (EXPO) Science Investigation Team' (15-WFIRST15-0004), administered by GSFC.

\section{DATA AVAILABILITY}

No new data were generated or analysed in support of this research.

\section{REFERENCES}

Abel T., Bryan G. L., Norman M. L., 2002, Science, 295, 93

Alvarez M. A., Ferraro S., Hill J. C., Hložek R., Ikape M., 2021, Phys. Rev. D, 103, 063518

Beckwith S. V. W. et al., 2006, AJ, 132, 1729

Behroozi P., Wechsler R. H., Hearin A. P., Conroy C., 2019, MNRAS, 488, 3143

Bouwens R. J. et al., 2015, ApJ, 803, 34 
Bouwens R. J. et al., 2021, AJ, 162, 47

Bowman J. D., Rogers A. E. E., Monsalve R. A., Mozdzen T. J., Mahesh N., 2018, Nature, 555, 67

Bradley R. F., Tauscher K., Rapetti D., Burns J. O., 2019, ApJ, 874, 153

Bromm V., 2013, Rep. Prog. Phys., 76, 112901

Bromm V., Larson R. B., 2004, ARA\&A, 42, 79

Cai Z. et al., 2011, ApJ, 736, L28

Cappelluti N. et al., 2013, ApJ, 769, 68

Chabrier G., 2003, PASP, 115, 763

Cooray A. et al., 2012a, Nature, 490, 514

Cooray A. et al., 2019, Bull. Am. Astron. Soc., 51, 23

Cooray A., Sheth R., 2002, Phys. Rep., 372, 1

Cooray A., Bock J. J., Keatin B., Lange A. E., Matsumoto T., 2004, ApJ, 606, 611

Cooray A., Gong Y., Smidt J., Santos M. G., 2012b, ApJ, 756, 92

Crosby B. D., O'Shea B. W., Smith B. D., Turk M. J., Hahn O., 2013, ApJ, 773, 108

DeBoer D. R. et al., 2017, PASP, 129, 045001

Dijkstra M., Kramer R., 2012, MNRAS, 424, 1672

Dopita M. A., Sutherland R. S., 2003, Astrophysics of the Diffuse Universe. Springer-Verlag, Berlin, Heidelberg

Doré O. et al., 2014, preprint (arXiv:1412.4872)

Draine B. T., Miralda-Escudé J., 2018, ApJ, 858, L10

Eldridge J. J., Stanway E. R., 2009, MNRAS, 400, 1019

Feng C., Cooray A., Bock J., Chang T.-C., Doré O., Santos M. G., Silva M. B., Zemcov M., 2019, ApJ, 875, 86

Ferland G. J. et al., 2013, Rev. Mex. Astron. Astrofis, 49, 137

Ferland G. J., 1980, PASP, 92, 596

Fernandez E. R., Komatsu E., 2006, ApJ, 646, 703

Fernandez E. R., Zaroubi S., 2013, MNRAS, 433, 2047 (FZ13)

Fernandez E. R., Komatsu E., Iliev I. T., Shapiro P. R., 2010, ApJ, 710, 1089

Fernandez E. R., Zaroubi S., Iliev I. T., Mellema G., Jelić V., 2014, MNRAS, 440, 298

Fialkov A., Barkana R., Tseliakhovich D., Hirata C. M., 2012, MNRAS, 424, 1335

Fialkov A., Barkana R., Visbal E., Tseliakhovich D., Hirata C. M., 2013, MNRAS, 432, 2909

Fialkov A., Barkana R., Pinhas A., Visbal E., 2014, MNRAS, 437, L36

Furlanetto S. R., 2021, MNRAS, 500, 3394

Furlanetto S. R., Loeb A., 2005, ApJ, 634, 1

Furlanetto S. R., Mirocha J., 2021, preprint (arXiv:2109.04488)

Furlanetto S. R., Mirocha J., Mebane R. H., Sun G., 2017, MNRAS, 472, 1576

Greif T. H., Springel V., White S. D. M., Glover S. C. O., Clark P. C., Smith R. J., Klessen R. S., Bromm V., 2011, ApJ, 737, 75

Grisdale K., Thatte N., Devriendt J., Pereira-Santaella M., Slyz A., Kimm T., Dubois Y., Yi S. K., 2021, MNRAS, 501, 5517

Grogin N. A. et al., 2011, ApJS, 197, 35

Haardt F., Madau P., 1996, ApJ, 461, 20

Haiman Z., Rees M. J., Loeb A., 1997, ApJ, 476, 458

Haiman Z., Abel T., Rees M. J., 2000, ApJ, 534, 11

Helgason K., Ricotti M., Kashlinsky A., 2012, ApJ, 752, 113

Helgason K., Ricotti M., Kashlinsky A., Bromm V., 2016, MNRAS, 455, 282

Heneka C., Cooray A., 2021, MNRAS, 506, 1573

Heneka C., Cooray A., Feng C., 2017, ApJ, 848, 52

Hills R., Kulkarni G., Meerburg P. D., Puchwein E., 2018, Nature, 564, E32

Holzbauer L. N., Furlanetto S. R., 2012, MNRAS, 419, 718

Hummel J. A., Stacy A., Jeon M., Oliveri A., Bromm V., 2015, MNRAS, 453,4136

Inayoshi K., Visbal E., Haiman Z., 2020, ARA\&A, 58, 27

Jaacks J., Thompson R., Finkelstein S. L., Bromm V., 2018, MNRAS, 475, 4396

Kashlinsky A., Arendt R., Gardner J. P., Mather J. C., Moseley S. H., 2004, ApJ, 608, 1

Kashlinsky A., Arendt R. G., Mather J., Moseley S. H., 2005, Nature, 438, 45
Kashlinsky A., Arendt R. G., Ashby M. L. N., Fazio G. G., Mather J., Moseley S. H., 2012, ApJ, 753, 63

Kashlinsky A., Mather J. C., Helgason K., Arendt R. G., Bromm V., Moseley S. H., 2015a, ApJ, 804, 99

Kashlinsky A., Arendt R. G., Atrio-Barandela F., Helgason K., 2015b, ApJ, 813, L12

Kashlinsky A., Arendt R. G., Atrio-Barandela F., Cappelluti N., Ferrara A., Hasinger G., 2018, Rev. Mod. Phys., 90, 025006

Knox L., 1995, Phys. Rev. D, 52, 4307

Lanz A. et al., 2014, in Oschmann Jacobus M. J., Clampin M., Fazio G. G., MacEwen H. A., eds, SPIE Conf. Ser. Vol. 9143, Space Telescopes and Instrumentation 2014: Optical, Infrared, and Millimeter Wave. SPIE, Bellingham, p. $91433 \mathrm{~N}$

Lewis A., Challinor A., Lasenby A., 2000, ApJ, 538, 473

Liu B., Bromm V., 2020, MNRAS, 497, 2839

Loeb A., Furlanetto S. R., 2013, The First Galaxies in the Universe. Princeton Univ. Press, Princeton, NJ

Lotz J. M. et al., 2017, ApJ, 837, 97

Madau P., 1995, ApJ, 441, 18

Madau P., Silk J., 2005, MNRAS, 359, L37

Maio U., Ciardi B., Dolag K., Tornatore L., Khochfar S., 2010, MNRAS, 407,1003

Mao X.-C., 2014, ApJ, 790, 148

Mas-Ribas L., Dijkstra M., 2016, ApJ, 822, 84

Mas-Ribas L., Dijkstra M., Forero-Romero J. E., 2016, ApJ, 833, 65

Mas-Ribas L., Hennawi J. F., Dijkstra M., Davies F. B., Stern J., Rix H.-W., 2017, ApJ, 846, 11

Mason C. A., Trenti M., Treu T., 2015, ApJ, 813, 21

McKee C. F., Tan J. C., 2008, ApJ, 681, 771

Mebane R. H., Mirocha J., Furlanetto S. R., 2018, MNRAS, 479, 4544

Mebane R. H., Mirocha J., Furlanetto S. R., 2020, MNRAS, 493, 1217

Mellema G. et al., 2013, Exp. Astron., 36, 235

Mészáros P., Rees M. J., 2010, ApJ, 715, 967

Mirocha J., 2014, MNRAS, 443, 1211

Mirocha J., Furlanetto S. R., 2019, MNRAS, 483, 1980

Mirocha J., Furlanetto S. R., Sun G., 2017, MNRAS, 464, 1365

Mirocha J., Mebane R. H., Furlanetto S. R., Singal K., Trinh D., 2018, MNRAS, 478, 5591

Mirocha J., La Plante P., Liu A., 2021, MNRAS, 507, 3872

Moster B. P., Somerville R. S., Maulbetsch C., van den Bosch F. C., Macciò A. V., Naab T., Oser L., 2010, ApJ, 710, 903

Nagao T., Motohara K., Maiolino R., Marconi A., Taniguchi Y., Aoki K., Ajiki M., Shioya Y., 2005, ApJ, 631, L5

Naoz S., Yoshida N., Gnedin N. Y., 2012, ApJ, 747, 128

O'Shea B. W., Norman M. L., 2007, ApJ, 654, 66

Oesch P. A., Bouwens R. J., Illingworth G. D., Labbé I., Stefanon M., 2018, ApJ, 855, 105

Oh S. P., Haiman Z., 2002, ApJ, 569, 558

Okamoto T., Gao L., Theuns T., 2008, MNRAS, 390, 920

Osterbrock D. E., Ferland G. J., 2006, Astrophysics of Gaseous Nebulae and Active Galactic Nuclei. University Science Books, Mill Valley, CA

Pagano L., Delouis J. M., Mottet S., Puget J. L., Vibert L., 2020, A\&A, 635, A99

Pallottini A., Ferrara A., Gallerani S., Salvadori S., D’Odorico V., 2014, MNRAS, 440, 2498

Park J., Gillet N., Mesinger A., Greig B., 2020, MNRAS, 491, 3891

Planck Collaboration XIII, 2016, A\&A, 594, A13

Qin Y., Poulin V., Mesinger A., Greig B., Murray S., Park J., 2020, MNRAS, 499,550

Qin Y., Mesinger A., Greig B., Park J., 2021, MNRAS, 501, 4748

Rahmati A., Schaye J., Bower R. G., Crain R. A., Furlong M., Schaller M., Theuns T., 2015, MNRAS, 452, 2034

Raiter A., Schaerer D., Fosbury R. A. E., 2010, A\&A, 523, A64

Ricotti M., 2016, MNRAS, 462, 601

Robertson B. E., Ellis R. S., Furlanetto S. R., Dunlop J. S., 2015, ApJ, 802, L19

Rydberg C.-E., Zackrisson E., Lundqvist P., Scott P., 2013, MNRAS, 429, 3658 
Safranek-Shrader C., Agarwal M., Federrath C., Dubey A., Milosavljević M., Bromm V., 2012, MNRAS, 426, 1159

Salvaterra R., Ferrara A., 2003, MNRAS, 339, 973

Santos M. R., Bromm V., Kamionkowski M., 2002, MNRAS, 336, 1082

Sarmento R., Scannapieco E., Cohen S., 2018, ApJ, 854, 75

Schaerer D., 2002, A\&A, 382, 28

Schaerer D., 2003, A\&A, 397, 527

Schauer A. T. P. et al., 2017, MNRAS, 467, 2288

Schauer A. T. P., Glover S. C. O., Klessen R. S., Clark P., 2021, MNRAS, 507,1775

Schauer A. T. P., Drory N., Bromm V., 2020, ApJ, 904, 145

Schneider A., Giri S. K., Mirocha J., 2021, Phys. Rev. D, 103, 083025

Scott B., Upton Sanderbeck P., Bird S., 2021, preprint (arXiv:2104.00017)

Seo H. J., Lee H. M., Matsumoto T., Jeong W. S., Lee M. G., Pyo J., 2015, ApJ, 807, 140

Sims P. H., Pober J. C., 2020, MNRAS, 492, 22

Skinner D., Wise J. H., 2020, MNRAS, 492, 4386

Sobral D., Matthee J., Darvish B., Schaerer D., Mobasher B., Röttgering H. J. A., Santos S., Hemmati S., 2015, ApJ, 808, 139

Spergel D. et al., 2015, preprint (arXiv:1503.03757)

Stacy A., Greif T. H., Bromm V., 2010, MNRAS, 403, 45

Stacy A., Greif T. H., Bromm V., 2012, MNRAS, 422, 290

Stark D. P., Schenker M. A., Ellis R., Robertson B., McLure R., Dunlop J., 2013, ApJ, 763, 129

Stecher T. P., Williams D. A., 1967, ApJ, 149, L29

Stefanon M., Bouwens R. J., Labbé I., Illingworth G. D., Gonzalez V., Oesch P. A., 2021, preprint (arXiv:2103.16571)

Steidel C. C., Erb D. K., Shapley A. E., Pettini M., Reddy N., Bogosavljević M., Rudie G. C., Rakic O., 2010, ApJ, 717, 289

Sugimura K., Matsumoto T., Hosokawa T., Hirano S., Omukai K., 2020, ApJ, 892, L14

Sun G. et al., 2021, ApJ, 915, 33

Sun G., Furlanetto S. R., 2016, MNRAS, 460, 417

Sun G., Hensley B. S., Chang T.-C., Doré O., Serra P., 2019, ApJ, 887, 142

Susa H., Hasegawa K., Tominaga N., 2014, ApJ, 792, 32
Tacchella S., Bose S., Conroy C., Eisenstein D. J., Johnson B. D., 2018, ApJ, 868,92

Tanaka T., Hasegawa K., 2021, MNRAS, 502, 463

Tegmark M., Silk J., Rees M. J., Blanchard A., Abel T., Palla F., 1997, ApJ, 474,1

Thomas R. M., Zaroubi S., 2008, MNRAS, 384, 1080

Toma K., Sakamoto T., Mészáros P., 2011, ApJ, 731, 127

Trac H., Cen R., Mansfield P., 2015, ApJ, 813, 54

Trenti M., Stiavelli M., 2009, ApJ, 694, 879

Trenti M., Stiavelli M., Shull J. M., 2009, ApJ, 700, 1672

Tseliakhovich D., Hirata C., 2010, Phys. Rev. D, 82, 083520

Tumlinson J., Shull J. M., 2000, ApJ, 528, L65

Turk M. J., Abel T., O’Shea B., 2009, Science, 325, 601

Visbal E., Haiman Z., Terrazas B., Bryan G. L., Barkana R., 2014, MNRAS, 445,107

Visbal E., Haiman Z., Bryan G. L., 2015, MNRAS, 450, 2506

Visbal E., Haiman Z., Bryan G. L., 2018, MNRAS, 475, 5246

Windhorst R. A. et al., 2018, ApJS, 234, 41

Wise J. H., Abel T., 2007, ApJ, 671, 1559

Wolcott-Green J., Haiman Z., Bryan G. L., 2011, MNRAS, 418, 838

Wu X., McQuinn M., Eisenstein D., Irsic V., 2021, preprint (arXiv:2105.08737)

Xu H., Norman M. L., O'Shea B. W., Wise J. H., 2016a, ApJ, 823, 140

Xu H., Wise J. H., Norman M. L., Ahn K., O’Shea B. W., 2016b, ApJ, 833 , 84

Yang Y. P., Wang F. Y., Dai Z. G., 2015, A\&A, 582, A7

Yue B., Ferrara A., Salvaterra R., Chen X., 2013a, MNRAS, 431, 383

Yue B., Ferrara A., Salvaterra R., Xu Y., Chen X., 2013b, MNRAS, 433, 1556

Yung L. Y. A., Somerville R. S., Finkelstein S. L., Popping G., Davé R., 2019, MNRAS, 483, 2983

Zemcov M. et al., 2014, Science, 346, 732

This paper has been typeset from a $\mathrm{T}_{\mathrm{E}} \mathrm{X} / \mathrm{LT} \mathrm{E} \mathrm{X}$ file prepared by the author. 